\title{
Unification in the Union of Disjoint Equational Theories: Combining Decision Procedures
}

Franz Baader, Klaus Schulz

November 1991

Deutsches Forschungszentrum für Künstliche Intelligenz $\mathrm{GmbH}$

Postfach 2080

D-6750 Kaiserslautern, FRG

Tel.: (+49 631) 205-3211/13

Fax: (+49 631) 205-3210
Stuhlsatzenhausweg 3

D-6600 Saarbrücken 11, FRG

Tel.: (+49 681) 302-5252

Fax: (+49 681) 302-5341 


\section{Deutsches Forschungszentrum für Künstliche Intelligenz}

The German Research Center for Artificial Intelligence (Deutsches Forschungszentrum für Künstliche Intelligenz, DFKI) with sites in Kaiserslautern und Saarbrücken is a non-profit organization which was founded in 1988 by the shareholder companies ADV/Orga, AEG, IBM, Insiders, Fraunhofer Gesellschaft, GMD, Krupp-Atlas, Mannesmann-Kienzle, Philips, Siemens and Siemens-Nixdorf. Research projects conducted at the DFKI are funded by the German Ministry for Research and Technology, by the shareholder companies, or by other industrial contracts.

The DFKI conducts application-oriented basic research in the field of artificial intelligence and other related subfields of computer science. The overall goal is to construct systems with technical knowledge and common sense which - by using Al methods - implement a problem solution for a selected application area. Currently, there are the following research areas at the DFKI:

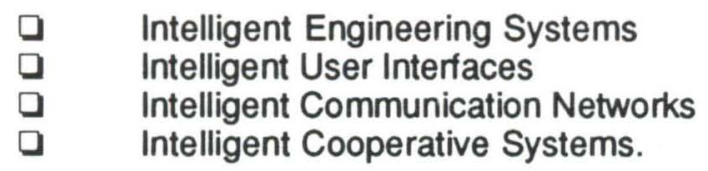

The DFKI strives at making its research results available to the scientific community. There exist many contacts to domestic and foreign research institutions, both in academy and industry. The DFKI hosts technology transfer workshops for shareholders and other interested groups in order to inform about the current state of research.

From its beginning, the DFKI has provided an attractive working environment for $\mathrm{AI}$ researchers from Germany and from all over the world. The goal is to have a staff of about 100 researchers at the end of the building-up phase.

Prof. Dr. Gerhard Barth

Director 
Unification in the Union of Disjoint Equational Theories:

Combining Decision Procedures

Franz Baader, Klaus Schulz

DFKI-RR-91-33 
This work has been supported by a grant from The Federal Ministry for Research and Technology (FKZ ITW-8903 0).

(C) Deutsches Forschungszentrum für Künstliche Intelligenz 1991

This work may not be copied or reproduced in whole or in part for any commercial purpose. Permission to copy in whole or in part without payment of fee is granted for nonprofit educational and research purposes provided that all such whole or partial copies include the following: a notice that such copying is by permission of Deutsches Forschungszentrum für Künstliche Intelligenz, Kaiserslautern, Federal Republic of Germany; an acknowledgement of the authors and individual contributors to the work; all applicable portions of this copyright notice. Copying, reproducing, or republishing for any other purpose shall require a licence with payment of fee to Deutsches Forschungszentrum für Künstliche Intelligenz. 


\title{
Unification in the Union of Disjoint Equational Theories: Combining Decision Procedures
}

Franz Baader

DFKI

Postfach 2080

6750 Kaiserslautern, Germany

baader@dfki.uni-kl.de
Klaus Schulz

CIS

University Munich, Leopoldstr. 139

8000 München 40, Germany

schulz@cis.uni-muenchen.dbp.de

\begin{abstract}
Most of the work on the combination of unification algorithms for the union of disjoint equational theories has been restricted to algorithms which compute finite complete sets of unifiers. Thus the developed combination methods usually cannot be used to combine decision procedures, i.e., algorithms which just decide solvability of unification problems without computing unifiers. In this paper we describe a combination algorithm for decision procedures which works for arbitrary equational theories, provided that solvability of so-called unification problems with constant restrictions-a slight generalization of unification problems with constants-is decidable for these theories. As a consequence of this new method, we can for example show that general $A$-unifiability, i.e., solvability of $A$-unification problems with free function symbols, is decidable. Here $A$ stands for the equational theory of one associative function symbol.

Our method can also be used to combine algorithms which compute finite complete sets of unifiers. Manfred Schmidt-SchauB' combination result, the until now most general result in this direction, can be obtained as a consequence of this fact. We also get the new result that unification in the union of disjoint equational theories is finitary, if general unification-i.e., unification of terms with additional free function symbols-is finitary in the single theories.
\end{abstract}




\section{Contents}

1 Introduction 3

2 Main results and consequences $\quad 7$

$\begin{array}{lll}3 & \text { The combination algorithm } & 10\end{array}$

4 Correctness of the combination algorithm $\quad 15$

5 Solving unification problems with constant restriction 21

5.1 Using algorithms for general unification . . . . . . . . . . 22

5.2 Using algorithms for constant elimination and for unification with constants ................. 27

6 Conclusion $\quad 29$ 


\section{Introduction}

$E$-unification is concerned with solving term equations modulo an equational theory $E$. The theory is called "unitary" ("finitary") if the solutions of a system of equations can always be represented by one (finitely many) solution(s). Otherwise the theory is of type "infinitary" or "zero" (see e.g., [Si89,JK90,Ba91] for an introduction to unification theory). Equational theories which are of unification type unitary or finitary play an important rôle in automated theorem provers with "built in" theories (see e.g., [P172,St85]), in generalizations of the Knuth-Bendix algorithm (see e.g., [JK86,Bc87]), and in logic programming with equality (see e.g., [JL84]). The reason is that these applications usually require algorithms which compute finite complete sets of unifiers, i.e., finite sets of unifiers from which all unifiers can be generated by instantiation. However, with the recent development of constraint approaches to theorem proving (see e.g., [Bü90]), term rewriting (see e.g., [KK89]), and logic programming (see e.g., [JL87,Co90]), the computation of finite complete sets of unifiers is no longer indespensable for these applications. It is enough to decide satisfiability of the constraints, that means e.g., solvability of the unification problems. In the present paper, the design of decision procedures for unification problems will be a major issue.

\section{The signature matters}

When considering unification in equational theories one has to be careful with regard to the signature over which the terms of the unification problems can be built. This leads to the distinction between elementary unification (where the terms to be unified are built over the signature of the equational theory, i.e., the function symbols occurring in the axioms of the theory), unification with constants (where additional free constant symbols may occur), and general unification (where additional free function symbols of arbitrary arity may occur).

The following facts show that there really is a difference between the three types of $E$-unification:

- There exist theories which are unitary with respect to elementary unification, but finitary with respect to unification with constants. An example for such a theory is the theory of abelian monoids, i.e., the theory of an associative-commutative $(A C)$ function symbol with a unit element (see e.g., [He87]). 
- There exists an equational theory for which elementary unification is decidable, but unification with constants is undecidable (see [Bü86]).

- From the development of the first algorithm for $A C$-unification with constants [St75,LS75] it took almost a decade until the termination of an algorithm for general $A C$-unification was shown by Fages [Fa84].

The applications of theory unification mentioned above require algorithms for general unification. This fact is illustrated by the following example.

Example 1.1 The theory $A=\{f(f(x, y), z)=f(x, f(y, z))\}$ only contains the binary symbol $f$. When talking about $A$-unification, one first thinks of unifying modulo $A$ terms built by using just the symbol $f$ and variables, or equivalently, of unifying words over the alphabet $V$ of all variables.

However, suppose that a resolution theorem prover-which has built in the theory $A$-gets the formula

$$
\exists x:(\forall y: f(x, y)=y \wedge \forall y \exists z: f(z, y)=x)
$$

as axiom. In a first step, this formula has to be Skolemized, i.e., the existential quantifiers have to be replaced by new function symbols. In our example, we need a nullary symbol $e$ and a unary symbol $i$ in the Skolemized form

$$
\forall y: f(e, y)=y \wedge \forall y: f(i(y), y)=e
$$

of the axiom. This shows that, even if we start with formulae containing only terms built over $f$, our theorem prover has to handle terms containing additional free symbols.

\section{The combination problem}

We have seen that the question of how algorithms for elementary unification (or for unification with constants) can be used to get algorithms for general unification is nontrivial and important for applications. Even more general, one often would like to derive algorithms for unification in the union of disjoint equational theories, i.e., in the union of several equational theories over disjoint signatures, from unification algorithms in the single theories. The importance for applications of this so-called "combination problem" is illustrated by the following example.

Example 1.2 Assume that we want to compute a canonical term rewriting system for the theory of Boolean rings. Thus we have a signature consisting 
of two binary symbols "+" and "*", a unary symbol "-", and two nullary symbols "0" and " 1 ". Since the addition and multiplication in Boolean rings is associative and commutative, and since commutativity cannot be oriented into a terminating rewrite rule, we have to use rewriting modulo associativity and commutativity of " + " and "*".

But then critical pairs also must be computed modulo associativity and commutativity of these two symbols. To be more precise, we consider the theories $A C_{+}:=\{(x+y)+z=x+(y+z), x+y=y+x\}$, and $A C_{*}:=$ $\{(x * y) * z=x *(y * z), x * y=y * x\}$. Critical pairs are computed with the help of general unification modulo $A C_{+} \cup A C_{*}$, i.e., modulo the union of the two disjoint equational theories $A C_{+}$and $A C_{*}$.

This example can also be used to demonstrate that going from elementary unification to general unification is in fact an instance of the combination problem. If we define the free theory for "-", " 0 " and "1" to be $F_{0,1,-}=$ $\{-x=-x, 0=0,1=1\}$, then one can use elementary unification modulo $A C_{+} \cup A C_{*} \cup F_{0,1,-}$ instead of general unification modulo $A C_{+} \cup A C_{*}$ for computing critical pairs.

When considering the combination problem, until now the attention was mostly restricted to finitary unifying theories, and by unification algorithm one meant a procedure which computes a finite complete set of unifiers. The problem was first considered in [St75,St81,Fa84,HS87] for the case where several $A C$-symbols and free symbols may occur in the terms to be unified. More general combination problems were, for example, treated in [Ki85, Ti86, He86,Ye87,BJ89], but the theories considered in these papers always had to satisfy certain restrictions (such as collapse-freeness or regularity ${ }^{1}$ ) on the syntactic form of their defining identities.

The problem was finally solved in its until now most general form by Schmidt-Schauß [Sc89]. His combination algorithm imposes no restriction on the syntactic form of the identities. The only requirements for a combination of disjoint theories $E, F$ are:

- All unification problems with constants must be finitary solvable in $E$ and $F$.

- All constant elimination problems must be finitary solvable in $E$ and $F$.

\footnotetext{
${ }^{1} \mathrm{~A}$ theory $E$ is called collapse-free if it does not contain an identity of the form $x=t$ where $x$ is a variable and $t$ is a non-variable term, and it is called regular if the left and right hand sides of the identities contain the same variables.
} 
A more efficient version of this combination algorithm has been described by Boudet [Bo90].

The method of Schmidt-Schauß can also handle theories which are not finitary. In this case, procedures which enumerate complete sets of unifiers for the single theories can be combined to a procedure enumerating a complete set of unifiers for their union. However, even if unification in the single theories is decidable, this does not show how to get a decision algorithm for unifiability in the combined theory.

The infinitary theory $A=\{f(f(x, y), z)=f(x, f(y, z))\}$ is an example for this case. In 1972, Plotkin [Pl72] has described a procedure which enumerates minimal complete sets of $A$-unifiers for general $A$-unification problems, and in 1977 Makanin [Ma77] has shown that $A$-unification with constants is decidable. But in 1991, decidability of general $A$-unification was still mentioned as an open problem by Kapur and Narendran [KN91] in their table of known decidability and complexity results for unification. Such a decision procedure could, for example, be useful when building associativity into a theorem prover via constraint resolution; and it could be used to make Plotkin's enumeration procedure terminating for equations having finite complete sets of $A$-unifiers.

In his paper on unification in the combination of arbitrary disjoint equational theories [Sc89], Schmidt-Schauß also treats the problem of how to combine decision procedures. But in this case he needs decision procedures for general unification in the single theories as prerequisites for his combination algorithm. Thus his result cannot be used to solve the above mentioned open problem of decidability of general $A$-unification.

The research which will be presented in this paper builds up on the ideas of Schmidt-Schauß and Boudet. It was motivated by the question of how to get a decision procedure for general $A$-unification. However, the results we have obtained are more general. We shall present a method which allows one to decide unifiability in the union of arbitrary disjoint equational theories, provided that solvability of so-called unification problems with constant restrictions - a slight generalization of unification problems with constantsis decidable for the single theories. In addition, our method can also be used to combine algorithms which compute finite complete sets of unifiers.

These main results and some of the interesting consequences will be described in the next section. Among these consequences are the new results that general $A$-unification is in fact decidable, and that the union of disjoint equational theories is finitary if the single theories are finitary with respect 
to general unification.

In Section 3 we shall present the combination algorithm for the decision problem, and describe how it can also be used to generate complete sets of unifiers. Section 4 proves the correctness of the method. In the fifth section we shall describe conditions under which algorithms for solving unification problems with constant restrictions exist. Some of the consequences mentioned in Section 2 depend on these results.

\section{Main results and consequences}

As mentioned in the introduction, we have to consider a slight generalization of $E$-unification problems with constants, so-called $E$-unification problems with constant restriction, which will be introduced below. Having an algorithm which solves these kind of problems is the only prerequisite necessary for our combination method.

Recall that an $E$-unification problem with constants is a finite set of equations $\Gamma=\left\{s_{1} \doteq t_{1}, \ldots, s_{n} \doteq t_{n}\right\}$, where the terms $s_{1}, \ldots, t_{n}$ are built from variables, the function symbols occurring in the axioms of $E$, and additional free constant symbols. Now, an E-unification problem with constant restriction is an ordinary $E$-unification problem with constants, $\Gamma$, where each free constant $c$ occurring in the problem $\Gamma$ is equipped with a set $V_{c}$ of variables, namely, the variables in whose image $c$ must not occur. A solution of the problem is an $E$-unifier $\sigma$ of $\Gamma$ such that for all $c, x$ with $x \in V_{c}$, the constant $c$ does not occur in $x \sigma$. Complete sets of solutions of unification problems with constant restriction are defined as in the case of ordinary unification problems.

It turns out that our combination method does not really need an algorithm which can handle $E$-unification problems with arbitrary constant restrictions; it is enough to deal with problems with a so-called linear constant restriction. Such a restriction is induced by a linear ordering on the variables and free constants as follows: Let $X$ be the set of all variables and $C$ be the set of all free constants occurring in $\Gamma$. For a given linear ordering $<$ on $X \cup C$, the sets $V_{c}$ are defined as $\{x \mid x$ is a variable with $x<c\}$.

We are now ready to formulate our first main result, which is concerned with combining decision algorithms. The combination algorithm which is used to establish this result will be described in the next section. 
Theorem 2.1 Let $E_{1}, \ldots, E_{n}$ be equational theories over disjoint signatures such that solvability of $E_{i}$-unification problems with linear constant restriction is decidable for $i=1, \ldots, n$. Then unifiability is decidable for the combined theory $E_{1} \cup \ldots \cup E_{n}$.

By "unifiability" we mean here solvability of elementary unification problems. However, we shall see below that the result can be lifted to general unification, and to solvability of unification problems with linear constant restriction. The theorem also has several other interesting consequences, which are listed below.

1. Let $E$ be an equational theory such that solvability of E-unification problems with linear constant restriction is decidable. Then solvability of general E-unification problems is decidable.

In fact, for a given set $\Omega$ of function symbols we can always build the free theory $F_{\Omega}$ as exemplified in Example 1.2. It is easy to see that $F_{\Omega}$ satisfies the assumption of the theorem; and obviously, any general unification problem modulo $E$ can be seen as an elementary unification modulo $E \cup F_{\Omega}$ (if $\Omega$ contains all the additional free function symbols occurring in the problem):

2. This argument also shows why the result of the theorem can be lifted to general unification: in order to get decidability of general unification modulo $E_{1} \cup \ldots \cup E_{n}$, apply the theorem to $E_{1}, \ldots, E_{n}, F_{\Omega}$.

3. General A-unifiability is decidable.

For $A$, decidability of unification problems with constant restriction is an easy consequence of a result by Schulz [Sh91] on a generalization of Makanin's procedure. This result shows that it is still decidable whether a given $A$-unification problem with constants has a solution for which the words substituted for the variables in the problem are elements of given regular languages over the constants. It is easy to see that problems with constant restriction are a special case of these more generally restricted problems.

4. General AI-unifiability, where $A I:=A \cup\{f(x, x)=x\}$, is decidable. This was also stated as an open problem in [KN91]. For $A I$, decidability of unification problems with constant restriction easily follows from the well-known fact (see e.g., [Ho76]) that finitely generated idempotent semigroups are finite. 
5. If solvability of the $E_{i}$-unification problems with linear constant restriction can be decided by an NP-algorithm, then unifiability in the combined theory is also NP-decidable.

This fact will become obvious once we have described our combination algorithm. As a consequence one gets easy proofs of Kapur and Narendran's results [KN91] that solvability of general $A C$ - and ACI-unification problems can be decided by NP-algorithms. For these theories, NPdecidability of unification problems with constant restriction can be shown very similarly as in the case of ordinary unification problems with constants.

6. Let $E_{1}, \ldots, E_{n}$ be equational theories over disjoint signatures such that solvability of general $E_{i}$-unification problems is decidable for $i=1, \ldots, n$. Then unifiability is decidable for the combined theory $E_{1} \cup \ldots \cup E_{n}$. This result, which was first proved by Schmidt-Schauß (see [Sc89], Theorem 10.6), can also be obtained as a corollary to our theorem. In fact, we can show that solvability of $E$-unification problems with linear constant restriction can be reduced to solvability of general $E$-unification problems (see Section 5).

7. Together with the second consequence mentioned above, this reduction also shows that the result of Theorem 2.1 can be lifted to unification problems with linear constant restriction.

The algorithm which will be introduced for proving Theorem 2.1 can also be used to compute complete sets of unifiers.

Theorem 2.2 Let $E_{1}, \ldots, E_{n}$ be equational theories over disjoint signatures such that all $E_{i}$-unification problems with linear constant restriction have finite complete set of solutions $(i=1, \ldots, n)$. Then the combined theory $E_{1} \cup \ldots \cup E_{n}$ is finitary.

Again, we are talking about elementary unification for the combined theory; but as for the case of the decision problem, the result can easily be lifted to general unification, and to unification problems with linear constant restriction. It should be noted that this result is effective in the sense that we really get an algorithm computing finite complete set of unifiers for the combined theory, provided that for the single theories there exist algorithms computing finite complete sets of solutions of unification problems with linear constant restriction. In the following, we mention two other interesting consequences of the theorem. 
8. Let $E_{1}, \ldots, E_{n}$ be equational theories over disjoint signatures which are finitary with respect to general unification. Then the combined theory $E_{1} \cup \ldots \cup E_{n}$ is finitary.

In fact, we can show how finite complete sets of unifiers for general $E_{i^{-}}$ unification problems can be used to construct finite complete sets of solutions for unification problems with linear constant restriction (see Section 5).

9. Algorithms which compute finite complete sets of unifiers for unification with constants, and finite complete sets of constant eliminators can be used to get an algorithm which computes finite complete sets of solutions for unification problems with constant restriction (see Section 5). As a consequence, the combination result of Schmidt-Schauß ([Sc89], Corollary 7.14) mentioned in the introduction can also be obtained as a corollary to Theorem 2.2.

\section{The combination algorithm}

For the sake of convenience we shall restrict the presentation to the combination of two theories. The combination of more than two theories can be treated analogously. Before we can start with the description of the algorithm we have to introduce some notation.

Let $E_{1}, E_{2}$ be two equational theories built over the disjoint signatures $\Omega_{1}, \Omega_{2}$, and let $E=E_{1} \cup E_{2}$ denote their union. Since we are only interested in elementary $E$-unification, we can restrict our attention to terms built from variables and symbols of $\Omega_{1} \cup \Omega_{2}$. The elements of $\Omega_{1}$ will be called 1-symbols and the elements of $\Omega_{2} 2$-symbols. A term $t$ is called $i$-term iff it is of the form $t=f\left(t_{1}, \ldots, t_{n}\right)$ for an $i$-symbol $f(i=1,2)$. A subterm $s$ of a 1-term $t$ is called alien subterm of $t$ iff it is a 2-term such that every proper superterm of $s$ in $t$ is a 1 -term. Alien subterms of 2 -terms are defined analogously. An $i$-term $s$ is pure iff it contains only $i$-symbols and variables. An equation $s \doteq t$ is pure iff there exists an $i, 1 \leq i \leq 2$, such that $s$ and $t$ are pure $i$-terms or variables; this equation is then called an $i$-equation. Please note that according to this definition equations of the form $x \doteq y$ where $x$ and $y$ are variables are both 1 - and 2-equations. In the following, the symbols $x, y, z$, with or without indices, will always stand for variables.

Example 3.1 Let $\Omega_{1}$ consist of the binary (infix) symbol "o" and $\Omega_{2}$ of the unary symbol " $h$ ", let $E_{1}:=\{x \circ(y \circ z)=(x \circ y) \circ z\}$ be the theory which 
says that "o" is associative, and let $E_{2}:=\{h(x)=h(x)\}$ be the free theory for " $h$ ".

The term $y \circ h(z \circ h(x))$ is a 1-term which has $h(z \circ h(x))$ as its only alien subterm. The equation $h\left(x_{1}\right) \circ x_{2} \doteq y$ is not pure, but it can be replaced by two pure equations as follows. We replace the alien subterm $h\left(x_{1}\right)$ of $h\left(x_{1}\right) \circ x_{2}$ by a new variable $z$. This yields the pure equation $z \circ x_{2} \doteq y$. In addition, we consider the new equation $z \doteq h\left(x_{1}\right)$. This process of replacing alien subterms by new variables is called variable abstraction. It will be the first of the five steps of our combination algorithm.

\section{The main procedure}

The input for the combination algorithm is an elementary $E$-unification problem, i.e., a system $\Gamma_{0}=\left\{s_{1} \doteq t_{1}, \ldots, s_{n} \doteq t_{n}\right\}$, where the terms $s_{1}, \ldots, t_{n}$ are built from variables and the function symbols occurring in $\Omega_{1} \cup \Omega_{2}$, the signature of $E=E_{1} \cup E_{2}$. The first two steps of the algorithm are deterministic, i.e., they transform the given system of equations into one new system.

Step 1: variable abstraction. Alien subterms are successively replaced by new variables until all terms occurring in the system are pure. To be more precise, assume that $s \doteq t$ or $t \doteq s$ is an equation in the current system, and that $s$ contains the alien subterm $s_{1}$. Let $x$ be a variable not occurring in the current system, and let $s^{\prime}$ be the term obtained from $s$ by replacing $s_{1}$ by $x$. Then the original equation is replaced by the two equations $s^{\prime} \doteq t$ and $x \doteq s_{1}$. This process has to be iterated until all terms occurring in the system are pure. It is easy to see that this can be achieved after finitely many iterations. Now all the terms in the system are pure, but there may still exist non-pure equations, consisting of a 1-term on one side and a 2 -term on the other side.

Step 2: split non-pure equations. Each non-pure equations of the form $s \doteq t$ is replaced by two equations $x \doteq s, x \doteq t$ where the $x$ are always new variables.

It is quite obvious that these two steps do not change solvability of the system. The result is a system which consists of pure equations. The third and the fourth step are nondeterministic, i.e., a given system is transformed into finitely many new systems. Here the idea is that the original system is solvable iff at least one of the new systems is solvable. 
Step 3: variable identification. Consider all possible partitions of the set of all variables occurring in the system. Each of these partitions yields one of the new systems as follows. The variables in each class of the partition are "identified" with each other by choosing an element of the class as representative, and replacing in the system all occurrences of variables of the class by this representative.

Step 4: choose ordering and theory indices. This step doesn't change a given system, it just adds some information which will be important in the next step. For a given system, consider all possible strict linear orderings $<$ on the variables of the system, and all mappings ind from the set of variables into the set of theory indices $\{1,2\}$. Each pair $(<$, ind $)$ yields one of the new systems obtained from the given one.

The last step is again deterministic. It splits each of the systems already obtained into a pair of pure systems.

Step 5: split systems. A given system $\Gamma$ is split into two systems $\Gamma_{1}$ and $\Gamma_{2}$ such that $\Gamma_{1}$ contains only 1 -equations and $\Gamma_{2}$ only 2 -equations. These systems can now be considered as unification problems with linear constant restriction. In the system $\Gamma_{i}$, the variables with index $i$ are still treated as variables, but the variables with alien index $j \neq i$ are treated as free constants. The linear constant restriction for $\Gamma_{i}$ is induced by the linear ordering chosen in the previous step.

The output of the algorithm is thus a finite set of pairs $\left(\Gamma_{1}, \Gamma_{2}\right)$ where the first component $\Gamma_{1}$ is an $E_{1}$-unification problem with linear constant restriction, and the second component $\Gamma_{2}$ is an $E_{2}$-unification problem with linear constant restriction.

Proposition 3.2 The input system $\Gamma_{0}$ is solvable if and only if there exists a pair $\left(\Gamma_{1}, \Gamma_{2}\right)$ in the output set such that $\Gamma_{1}$ and $\Gamma_{2}$ are solvable.

A proof of this proposition is described in the next section. Obviously, if solvability of $E_{1}$ - and $E_{2}$-unification problems with linear constant restrictions is decidable, the proposition implies decidability of elementary $E$ unifiability, which proves Theorem 2.1. 


\section{An example}

We consider the theories $E_{1}$ and $E_{2}$ of Example 3.1, and the unification problem

$$
\left\{h(x) \circ y=y \circ h\left(z_{1} \circ z_{2}\right)\right\} .
$$

Step 1: variable abstraction. This step results in the new system

$$
\left\{x_{1} \circ y=y \circ x_{2}, x_{1}=h(x), x_{2}=h\left(x_{3}\right), x_{3}=z_{1} \circ z_{2}\right\} .
$$

Step 2: split non-pure equations. Since all equations are already pure, nothing is done in this step.

Step 3: variable identification. As an example, we consider the partition where $x_{1}$ and $x_{2}$ are in one class, and all the other variables are in singleton classes. Choosing $x_{1}$ as representative for its class, we obtain the new system

$$
\left\{x_{1} \circ y=y \circ x_{1}, x_{1}=h(x), x_{1}=h\left(x_{3}\right), x_{3}=z_{1} \circ z_{2}\right\} .
$$

Step 4: choose ordering and theory indices. As an example, we take the linear ordering

$$
z_{1}<z_{2}<x_{3}<x<x_{1}<y
$$

and the theory indices

$$
\operatorname{ind}\left(x_{1}\right)=\operatorname{ind}(x)=\operatorname{ind}\left(z_{1}\right)=\operatorname{ind}\left(z_{2}\right)=2 \text { and } \operatorname{ind}\left(x_{3}\right)=\operatorname{ind}(y)=1 .
$$

Step 5: split systems. On the one hand, we get the system

$$
\Gamma_{1}=\left\{x_{1} \circ y=y \circ x_{1}, x_{3}=z_{1} \circ z_{2}\right\}
$$

consisting of pure 1-equations. In this system the variables with index 1, i.e., $x_{3}$ and $y$, are still treated as variables, but the variables of index 2, i.e., $x_{1}, z_{1}$ and $z_{2}$, are treated as free constants. The linear constant restriction induced by the linear ordering is given by $V_{x_{1}}=\left\{x_{3}\right\}, V_{z_{1}}=$ $V_{z_{2}}=\emptyset$.

On the other hand, we obtain the system

$$
\Gamma_{2}=\left\{x_{1}=h(x), x_{1}=h\left(x_{3}\right)\right\}
$$

consisting of pure 2-equations. Here $x$ and $x_{1}$ are treated as variables, and $x_{3}$ is treated as free constant. The constant restriction is given by $V_{x_{3}}=\emptyset$. 
This pair $\left(\Gamma_{1}, \Gamma_{2}\right)$ is one element in the set which is the output of the algorithm. It is easy to see that $\Gamma_{1}$ has the solution $\left\{x_{3} \mapsto z_{1} \circ z_{2}, y \mapsto x_{1}\right\}$, and $\Gamma_{2}$ has the solution $\left\{x_{1} \mapsto h\left(x_{3}\right), x \mapsto x_{3}\right\}$. Consequently, the proposition implies that the original system has a solution.

\section{Combination of unifiers}

The combination algorithm can also be used to compute complete sets of unifiers for elementary $\left(E_{1} \cup E_{2}\right)$-unification problems, provided that one can compute finite complete sets of solutions for all $E_{i}$-unification problems with linear constant restriction $(i=1,2)$. The reason is that solutions of the problems $\Gamma_{1}, \Gamma_{2}$ in the output of the algorithm can be combined to solutions of the original input system. This combined solution is defined inductively over the linear ordering chosen in Step 4 of the algorithm.

Assume that $\sigma_{1}$ is a solution of $\Gamma_{1}$ and $\sigma_{2}$ is a solution of $\Gamma_{2}$. Without loss of generality we may assume that the substitution $\sigma_{i}$ maps all variables of index $i$ to terms containing only variables of index $j \neq i$ (which are treated as free constants in $\Gamma_{i}$ ) or new variables, i.e., variables not occurring in $\Gamma_{0}$, $\Gamma_{1}$, or $\Gamma_{2}$. This can simply be achieved by renaming variables if necessary. First, we define the combined solution $\sigma$ on the variables occurring in the system obtained after Step 4 of the algorithm. Note that the input system $\Gamma_{0}$ may contain additional variables which have been replaced during the variable identification step.

Let $x$ be the least variable with respect to the linear ordering chosen in Step 4, and let $i$ be its index. Since the solution $\sigma_{i}$ of $\Gamma_{i}$ satisfies the constant restriction induced by the linear ordering, the term $x \sigma_{i}$ does not contain any variables of index $j \neq i$ (Recall that these variables are treated as free constants in $\Gamma_{i}$.) Thus we can simply define $x \sigma:=x \sigma_{i}$.

Now let $x$ be an arbitrary variable with index $i$, and let $y_{1}, \ldots, y_{m}$ be the variables with index $j \neq i$ occurring in $x \sigma_{i}$. Since $\sigma_{i}$ satisfies the constant restriction induced by the linear ordering, the variables $y_{1}, \ldots, y_{m}$ (which are treated as free constants in $\Gamma_{i}$ ) have to be smaller than $x$. That means that $y_{1} \sigma, \ldots, y_{m} \sigma$ are already defined. The term $x \sigma$ is now obtained from $x \sigma_{i}$ by replacing the $y_{k}$ by $y_{k} \sigma(k=1, \ldots, m)$. Because we have assumed that the other variables occurring in $x \sigma_{i}$ are new variables, we thus have $x \sigma=x \sigma_{i} \sigma$.

Finally, let $x$ be a variable of the input system which has been replaced by the variable $y$ during the variable identification step. Thus $y \sigma$ is already defined, and we can simply set $x \sigma:=y \sigma$. 
For all variables $z$ not occurring in the input system, or in $\Gamma_{1}$ or $\Gamma_{2}$, we define $z \sigma:=z$.

Example 3.3 For the above example, the solutions $\sigma_{1}=\left\{x_{3} \mapsto z_{1} \circ z_{2}, y \mapsto\right.$ $\left.x_{1}\right\}$ and $\sigma_{2}=\left\{x_{1} \mapsto h\left(x_{3}\right), x \mapsto x_{3}\right\}$ of $\Gamma_{1}, \Gamma_{2}$ are combined to $\left\{z_{1} \mapsto z_{1}, z_{2} \mapsto\right.$ $\left.z_{2}, x_{3} \mapsto z_{1} \circ z_{2}, x \mapsto z_{1} \circ z_{2}, x_{1} \mapsto h\left(z_{1} \circ z_{2}\right), x_{2} \mapsto h\left(z_{1} \circ z_{2}\right), y \mapsto h\left(z_{1} \circ z_{2}\right)\right\}$.

This construction can now be used to generate complete sets of unifiers for elementary $\left(E_{1} \cup E_{2}\right)$-unification problems. For a given input system $\Gamma_{0}$, let $\left\{\left(\Gamma_{1,1}, \Gamma_{1,2}\right), \ldots,\left(\Gamma_{n, 1}, \Gamma_{n, 2}\right)\right\}$ be the output of the combination algorithm. For $i=1, \ldots, n$ and $j=1,2$, let $M_{i, j}$ be a complete set of solutions of the $E_{i}$-unification problem with linear constant restriction, $\Gamma_{i, j}$.

Proposition 3.4 The set of substitutions

$$
\bigcup_{i=1}^{n}\left\{\sigma \mid \sigma \text { is the combined solution obtained from } \sigma_{1} \in M_{i, 1} \text { and } \sigma_{2} \in M_{i, 2}\right\}
$$

is a complete set of $\left(E_{1} \cup E_{2}\right)$-unifiers of the input system $\Gamma_{0}$.

A proof of this proposition will be given in the next section. Obviously, if all the sets $M_{i, j}$ are finite, then the complete set given by the proposition is also finite, which proves Theorem 2.2.

\section{Correctness of the combination algorithm}

In this section we shall prove Proposition 3.2 and Proposition 3.4, which shows that our combination method is correct when applied to decision problems. Before we can start with our task, we have to introduce a useful tool, which has first been utilized in connection with the combination problem in [BJ89], namely unfailing completion of the combined theory.

Let $E_{1}, E_{2}$ be equational theories over disjoint signatures $\Omega_{1}, \Omega_{2}$. We assume that both theories are consistent, that means, they have at least one model of cardinality greater than one, or equivalently, the identity $x={ }_{E_{i}} y$ does not hold in either theory. One can now apply unfailing completion (see e.g., [DJ87] for definitions and properties) to the combined theory $E=$ $E_{1} \cup E_{2}$. This yields a possibly infinite ordered-rewriting system $R$ which is confluent and terminating on ground terms. In the following, we shall also apply this system to terms containing variables from a fixed countable set of 
variables $X_{0}$; but this is not a problem because these variables can simply be treated like constants. In particular, this means that the simplification ordering used during the completion must also take care of these additional "constants." The ordered-rewriting system $R$ consists of (possibly infinitely many) equations $g=d$. Such an equation can be applied to a term $s \in$ $T\left(\Omega_{1} \cup \Omega_{2}, X_{0}\right)$ iff there exists an occurrence $u$ in $t$ and a substitution $\tau$ such that $s=s[u \leftarrow g \tau](s=s[u \leftarrow d \tau]$, resp.) and $g \tau$ is greater than $d \tau(d \tau$ is greater than $g \tau$, resp.) with respect to the simplification ordering. This application results in the new term $s[u \leftarrow d \tau](s[u \leftarrow g \tau]$, resp.).

It is easy to see that, because the signatures of $E_{1}$ and $E_{2}$ are disjoint, the system $R$ is the union of two systems $R_{1}$ and $R_{2}$, where the terms in $R_{i}$ are built over the signature $\Omega_{i}(i=1,2)$. The $R_{i}$ is just the system which would be obtained by applying unfailing completion to $E_{i}$. This is an easy consequence of the definition of critical pairs used for unfailing completion, and of the fact that $E_{1}$ and $E_{2}$ are assumed to be consistent.

Let $T\left(\Omega_{1} \cup \Omega_{2}, X_{0}\right)$ be the set of terms built from function symbols in $\Omega_{1} \cup \Omega_{2}$ and variables in $X_{0}$, and let $T_{\downarrow R}$ denote its $R$-irreducible elements. We consider an arbitrary bijection $\pi: T_{\downarrow R} \longrightarrow Y$ where $Y$ is a set of variables which is disjoint to $X_{0}$. This bijection induces mappings $\pi_{1}, \pi_{2}$ of terms in $T\left(\Omega_{1} \cup \Omega_{2}, X_{0}\right)$ to terms in $T\left(\Omega_{1} \cup \Omega_{2}, Y\right)$ as follows. For variables $x \in X_{0}, x^{\pi_{1}}:=\pi(x)$ (Note that variables are always $R$-irreducible.) If $t=f\left(t_{1}, \ldots, t_{n}\right)$ for a 1 -symbol $f$, then $t^{\pi_{1}}:=f\left(t_{1}^{\pi_{1}}, \ldots, t_{n}^{\pi_{1}}\right)$. Finally, if $t$ is a 2 -term then $t^{\pi_{1}}:=y$ where $y=\pi(s)$ for the unique $R$-irreducible element $s$ of the $=_{E}$-class of $t$. The mapping $\pi_{2}$ is defined analogously. The mappings $\pi_{i}$ may be regarded as projections which map a possibly mixed term to an $i$ pure term. We write these mappings as superscripts to distinguish them from substitutions. The inverse $\pi^{-1}$ of $\pi$ can be seen as a substitution which map the variables $y$ in $Y$ back to the terms $\pi^{-1}(y)$, and is the identity on all other variables. Obviously, we have $t^{\pi_{i}} \pi^{-1}=_{E} t$ for all terms $t \in T\left(\Omega_{1} \cup \Omega_{2}, X_{0}\right)$, and if $t$ is an $R$-irreducible term or an $i$-term such that all its alien subterms are $R$-irreducible, then $\left(t^{\pi_{i}}\right) \pi^{-1}=t$.

A substitution $\sigma$ is called $R$-normalized on a finite set of variables $Z$ iff $z \sigma \in T_{\downarrow R}$ for all variables $z \in Z$. The next lemma will be important in the proof of Proposition 3.2.

Lemma 4.1 Let $s, t$ be pure $i$-terms or variables, and let $\sigma$ be a substitution which is $R$-normalized on the variables occurring in $s, t$. Then

$$
s \sigma={ }_{E} t \sigma \quad \text { iff } \quad(s \sigma)^{\pi_{i}}=E_{i}(t \sigma)^{\pi_{i}} .
$$


Proof. (1) The if-direction is easy to prove. Obviously, $(s \sigma)^{\pi_{i}}=E_{E_{i}}(t \sigma)^{\pi_{i}}$ implies $(s \sigma)^{\pi_{i}}={ }_{E}(t \sigma)^{\pi_{i}}$, and thus $(s \sigma)^{\pi_{i}} \pi^{-1}={ }_{E}(t \sigma)^{\pi_{i}} \pi^{-1}$. By our assumptions on $s, t$ and $\sigma$, the $j$-terms (for $j \neq i$ ) in $s \sigma$ and $t \sigma$ are $R$-irreducible, which finally yields $s \sigma=(s \sigma)^{\pi_{i}} \pi^{-1}={ }_{E}(t \sigma)^{\pi_{i}} \pi^{-1}=t \sigma$.

(2) From $s \sigma={ }_{E} t \sigma$ follows the existence of an $R$-irreducible term $r$ which is a common $R$-descendant of $s \sigma$ and $t \sigma$. Let us now consider the derivation $s_{0}:=s \sigma \rightarrow_{R} s_{1} \rightarrow_{R} \ldots r$ more closely. The goal is to show $s_{0}^{\pi_{i}}={ }_{E_{i}} s_{1}^{\pi_{i}}=E_{E_{i}}$ $\ldots r^{\pi_{i}}$. Symmetrically, we could then also deduce $(t \sigma)^{\pi_{i}}={ }_{E_{i}} r^{\pi_{i}}$, which finally would prove the lemma.

The case where $s$ is a variable is trivial since then $s_{0}$ is $R$-irreducible, which yields $s_{0}=r$. Thus assume that $s$ is a pure $i$-term. Since all alien subterms of $s \sigma$ are $R$-irreducible, the first step of the derivation from $s_{0}$ to $r$ must take place at an occurrence $u$ which is not inside an alien subterm of $s_{0}=s \sigma$. In particular, this means that it is done by applying a rule $g=d$ of $R_{i}$. To be more precise, there exists a substitution $\tau$ such that $s_{0}=s_{0}[u \leftarrow g \tau], s_{1}=s_{0}[u \leftarrow d \tau]$, and $g \tau$ is greater than $d \tau$ with respect to the simplification ordering. From the fact that $u$ is not inside an alien subterm of $s_{0}$ we get that $s_{0}^{\pi_{i}}=s_{0}^{\pi_{i}}\left[u \leftarrow(g \tau)^{\pi_{i}}\right]$ and $s_{1}^{\pi_{i}}=s_{0}^{\pi_{i}}\left[u \leftarrow(d \tau)^{\pi_{i}}\right]$.

In order to conclude $s_{0}^{\pi_{i}}={ }_{E_{i}} s_{1}^{\pi_{i}}$, it thus remains to be shown that $(g \tau)^{\pi_{i}}={ }_{E_{i}}(d \tau)^{\pi_{i}}$. To see this, we define the substitution $\tau^{\pi_{i}}:=\{x \mapsto$ $(x \tau)^{\pi_{i}} \mid x$ occurs in $g$ or $\left.d\right\}$. Since $g, d$ are pure $i$-terms or variables, we have $g\left(\tau^{\pi_{i}}\right)=(g \tau)^{\pi_{i}}$ and $d\left(\tau^{\pi_{i}}\right)=(d \tau)^{\pi_{i}}$. Because $g=d \in R_{i}$ implies $g={ }_{E_{i}} d$, we thus get $(g \tau)^{\pi_{i}}=g\left(\tau^{\pi_{i}}\right)={ }_{E_{i}} d\left(\tau^{\pi_{i}}\right)=(d \tau)^{\pi_{i}}$.

If we want to continue by induction, we have to know that all alien subterms of $s_{1}$ are $R$-irreducible. This need not be the case for arbitrary derivations from $s \sigma$ to $r$. The problem is that we only have an ordered-rewriting system which is terminating on ground terms. For this reason it may well be the case that $d$ contains variables not contained in $g$; and in general we cannot be sure that the image of these variables under $\tau$ does not introduce reducible alien subterms into $s_{1}$. However, if we assume that the derivation from $s \sigma$ to $r$ is a bottom-up derivation where all the matching substitutions (such as our $\tau$ ) are $R$-normalized, then $\tau$ cannot introduce reducible alien subterms. This assumption can be made without loss of generality because it is easy to see that, whenever a term is not $R$-irreducible, then we can apply a rule of $R$ to this term in a way that satisfies the constraints of the assumption. 


\section{Proof of Proposition 3.2}

First, we shall show soundness of the combination algorithm, that means, we have to demonstrate that $\Gamma_{0}$ is solvable if there exists a pair $\left(\Gamma_{1}, \Gamma_{2}\right)$ in the output set such that $\Gamma_{1}$ and $\Gamma_{2}$ are solvable.

Assume that $\sigma_{1}$ is a solution of $\Gamma_{1}$ and $\sigma_{2}$ is a solution of $\Gamma_{2}$. In the previous section we have already described how these two solutions of the single problems can be combined to a substitution $\sigma$, which we have called the combined solution. It remains to be shown that $\sigma$ is in fact a solution of $\Gamma_{0}$. Obviously, it is sufficient to prove that $\sigma$ is a solution of the system $\Gamma^{\prime}$ which was obtained by Step 4 of the algorithm, and which in Step 5 was split into $\Gamma_{1}$ and $\Gamma_{2}$. Let $s \doteq t$ be an equation in $\Gamma^{\prime}$, and assume without loss of generality that this equation was put into $\Gamma_{1}$ in Step 5 . Thus we know that $s \sigma_{1}={ }_{E_{1}} t \sigma_{1}$. As an easy consequence of the definition of $\sigma$, one gets that $\sigma=\sigma_{1} \sigma$. Since $s \sigma_{1}={ }_{E_{1}} t \sigma_{1}$ obviously implies $s \sigma_{1} \sigma={ }_{E_{1}} t \sigma_{1} \sigma$, and thus also $s \sigma_{1} \sigma={ }_{E} t \sigma_{1} \sigma$, this shows that $s \sigma={ }_{E} t \sigma$.

In the second part of the proof we have to show completeness of the combination algorithm, that means, we have to demonstrate that there exists a pair $\left(\Gamma_{1}, \Gamma_{2}\right)$ in the output set such that $\Gamma_{1}$ and $\Gamma_{2}$ are solvable if $\Gamma_{0}$ is solvable.

Let $\sigma$ be a solution of $\Gamma_{0}$. Without loss of generality we assume that $\sigma$ is also a solution of the system obtained after the first two steps of the algorithm, that the set $Y_{0}$ of all variables occurring in this system is disjoint to $X_{0}$, and that $\sigma$ is $R$-normalized on $Y_{0}$. In particular, this implies that the variables occurring in $y \sigma$ for $y \in Y_{0}$ are elements of $X_{0}$. The solution $\sigma$ can be used to define the correct alternatives in the nondeterministic steps of the combination algorithm:

- The partition of the set of all variables, which has to be chosen in the third step, is defined as follows. Two variables $y$ and $z$ are in the same class iff $y \sigma=z \sigma$. Obviously, this means that $\sigma$ is also a solution of the system obtained after the variable identification step corresponding to this partition.

- In the fourth step, the variable $y$ gets index $i$ if $y \sigma$ is an $i$-term. If $y \sigma$ is itself a variable, $y$ gets index 1 (This is arbitrary, we could have taken index 2 as well.)

- In the fourth step, we also have to choose an appropriate linear ordering on the variables occurring in the system. Consider the strict partial 
ordering defined by $y<z$ iff $y \sigma$ is a strict subterm of $z \sigma$. We take an arbitrary extension of this partial ordering to a linear ordering on the variables occurring in the system.

The choices we have just described determine a system $\Gamma^{\prime}$ in the set of systems obtained after Step 4 of the algorithm, and thus a particular pair of systems $\left(\Gamma_{1}, \Gamma_{2}\right)$ in the output set of the combination algorithm. It remains to be shown that $\Gamma_{1}, \Gamma_{2}$ are solvable. In order to define solutions $\sigma_{i}$ of these systems, we consider a bijection $\pi$ from the $R$-irreducible elements of $T\left(\Omega_{1} \cup \Omega_{2}, X_{0}\right)$ onto a set of variables $Y$.

This bijection has to satisfy two conditions. First, $Y$ should contain all the variables occurring in $\Gamma^{\prime}$. Since $\sigma$ is assumed to be $R$-normalized on $Y_{0}$, we have that $y \sigma$ is $R$-irreducible for all variables $y$ occurring in $\Gamma^{\prime}$. The second condition on $\pi$ is that $\pi(y \sigma)=y$ for all these variables $y$. For the satisfiability of these conditions, the variable identification step is important. The reason is that only because of this step we can be sure that $\Gamma^{\prime}$ does not contain two different variables $y, y^{\prime}$ with $y \sigma=y^{\prime} \sigma$.

As described above, the bijection $\pi$ induces mappings $\pi_{1}, \pi_{2}$. These mappings will now be used to construct the solutions $\sigma_{i}, i=1,2$. The substitution $\sigma_{i}$ is defined on the variables $y$ occurring in $\Gamma^{\prime}$ by $y \sigma_{i}:=(y \sigma)^{\pi_{i}}$.

If $y$ is a variable of index $j \neq i$, the term $y \sigma$ is either a variable in $X_{0}$ or a $j$-term. In both cases we get $y \sigma_{i}=(y \sigma)^{\pi_{i}}=\pi(y \sigma)=y$ by definition of $\sigma_{i}$ and $\pi_{i}$. This shows that $\sigma_{i}$ really treats the variables of index $j$ as constants.

Now assume that $s \doteq t$ is an equation in $\Gamma_{i}$. Since this equation is also contained in $\Gamma^{\prime}$, and since $\sigma$ solves $\Gamma^{\prime}$, we know that $s \sigma={ }_{E} t \sigma$. Since $\sigma$ was assumed to be $R$-normalized on $Y_{0}$, and since $s \doteq t$ is an $i$-equation, we can apply the lemma to get $(s \sigma)^{\pi_{i}}={ }_{E_{i}}(t \sigma)^{\pi_{i}}$. Using the definition of $\sigma_{i}$ and the fact that $s \doteq t$ is an $i$-equation, it is easy to see that $(s \sigma)^{\pi_{i}}=s \sigma_{i}$ and $(t \sigma)^{\pi_{i}}=t \sigma_{i}$. Thus $\sigma_{i}$ really solves the equation $s \doteq t$.

It remains to be shown that $\sigma_{i}$ satisfies the constant restriction. Assume that $x$ is a variable of index $i$, and that the variable $y$ of index $j \neq i$ (which is treated as a constant in $\Gamma_{i}$ ) occurs in $x \sigma_{i}$. We have to show that $x$ is not an element of $V_{y}$, i.e., that $x \nless y$. Recall that $x \sigma_{i}=(x \sigma)^{\pi_{i}}$, and that $x \sigma$ is $R$-irreducible. Thus, since $y \notin X_{0}$, the occurrence of $y$ in $x \sigma_{i}$ must come from the occurrence of $y \sigma$ as a subterm of $x \sigma$. Because of the identification step, the fact that $x$ and $y$ are different variables also implies that $x \sigma$ and $y \sigma$ are different terms. Thus $y \sigma$ is a strict subterm of $x \sigma$, which yields $y<x$ because of the way the linear ordering was chosen. 


\section{Proof of Proposition 3.4}

In the first part of the proof of Proposition 3.2 we have already shown that the elements of the set of substitution defined in the formulation of Proposition 3.4 are solutions of $\Gamma_{0}$. It remains to be shown that this set is complete.

Let $\tau$ be a solution of $\Gamma_{0}$. Without loss of generality we assume that $\tau$ is also a solution of the system obtained after the first two steps of the algorithm, that the set $Y_{0}$ of all variables occurring in this system is disjoint to $X_{0}$, and that $\tau$ is $R$-normalized on $Y_{0}$. In the second part of the proof of Proposition 3.2 we have shown that $\tau$ can be used to find a pair of systems $\left(\Gamma_{1}, \Gamma_{2}\right)$ in the output set of the combination algorithm, and to construct solutions $\tau_{1}$ and $\tau_{2}$ of these systems. This construction makes use of a bijection $\pi$ and mappings $\pi_{1}, \pi_{2}$ induced by this bijection as described above.

Since $\tau_{i}$ is a solution of $\Gamma_{i}$, there exist an element $\sigma_{i}$ in the complete set of solutions of $\Gamma_{i}$ and a substitution $\lambda_{i}$ such that $\tau_{i}={ }_{E_{i}} \sigma_{i} \lambda_{i}\left\langle Y_{i}\right\rangle,{ }^{2}$ where $Y_{i}$ denotes the set of variables occurring in $\Gamma_{i}$ (i.e., the variables of index $i$ ). Without loss of generality we may assume that the substitution $\sigma_{i}$ maps the variables in $Y_{i}$ to terms containing only variables of index $j \neq i$ (which are treated as constants by $\lambda_{i}$ ) or new variables from a set of variables $Z_{i}$. We may assume that the domain of $\lambda_{i}$ is $Z_{i}$, and that the sets $X_{0}, Y_{0}, Z_{1}, Z_{2}$ are pairwise disjoint.

As described in the first part of the proof of Proposition 3.2, the solutions $\sigma_{1}, \sigma_{2}$ of $\Gamma_{1}, \Gamma_{2}$ can be combined to a solution $\sigma$ of $\Gamma$. Since this combined solution is an element of the set of substitution defined in the formulation of Proposition 3.4, it remains to be shown that there exists a substitution $\lambda$ such that $\tau={ }_{E} \sigma \lambda\langle X\rangle$, where $X$ denotes the set of variables occurring in $\Gamma_{0}$. We define $\lambda:=\left(\lambda_{1} \cup \lambda_{2}\right) \pi^{-1}$, where $\left(\lambda_{1} \cup \lambda_{2}\right)$ is meant to denote the substitution which is equal to $\lambda_{i}$ on $Z_{i}(i=1,2)$, and the identity on all variables not contained in $Z_{1} \cup Z_{2}$.

First, we show $\tau={ }_{E} \sigma \lambda\left\langle Y_{1} \cup Y_{2}\right\rangle$. The proof is by induction on the linear ordering $<$ chosen in Step 4 of the combination algorithm. Without loss of generality, we consider a variable $y \in Y_{1}$. By the definition of $\sigma$, we have $y \sigma \lambda=\left(y \sigma_{1}\right) \sigma \lambda$. The variables occurring in the term $y \sigma_{1}$ are either variables of index 2, i.e., elements of $Y_{2}$, or new variables, i.e., elements of $Z_{1}$. We want to show that on these variables, the substitutions $\sigma \lambda$ and $\lambda_{1} \pi^{-1}$ coincide modulo $E$.

\footnotetext{
${ }^{2}$ Recall that, for a finite set $Z$ of variables, $\delta_{1}={ }_{E} \delta_{2}\langle Z\rangle$ means that $z \delta_{1}={ }_{E} z \delta_{2}$ for all $z \in Z$.
} 
Let $z_{1}$ be an element of $Z_{1}$ occurring in $y \sigma_{1}$. Since we have assumed that the elements of $Z_{1}$ are new variables, $z_{1}$ is not in the domain of $\sigma$, which yields $z_{1} \sigma \lambda=z_{1} \lambda$. By definition of $\lambda$, and since $z_{1} \in Z_{1}$, we get $z_{1} \lambda=z_{1} \lambda_{1} \pi^{-1}$.

If $y$ is the least variable with respect to the linear ordering $<$, then the term $y \sigma_{1}$ does not contain a variable of $Y_{2}$. This is so because $\sigma_{1}$ satisfies the linear constant restriction induced by $<$. Now assume that $y$ is an arbitrary variable in $Y_{1}$, and let $y_{2}$ be an element of $Y_{2}$ occurring in $y \sigma_{1}$. Since $\sigma_{1}$ satisfies the linear constant restriction, we know that $y_{2}<y$. By induction, we thus get $y_{2} \sigma \lambda={ }_{E} y_{2} \tau$. We also have $y_{2} \tau=\left(y_{2} \tau\right)^{\pi_{1}} \pi^{-1}=y_{2} \tau_{1} \pi^{-1}$. Since $\tau_{1}$ and $\lambda_{1}$ treat variables of index 2 as constants, we get $y_{2} \tau_{1} \pi^{-1}=y_{2} \pi^{-1}=$ $y_{2} \lambda_{1} \pi^{-1}$. Thus we have shown $y_{2} \sigma \lambda={ }_{E} y_{2} \lambda_{1} \pi^{-1}$.

To sum up, we have just shown that, for all variables $z$ occurring in $y \sigma_{1}$, we have $z \sigma \lambda={ }_{E} z \lambda_{1} \pi^{-1}$. Consequently, we get $y \sigma \lambda=\left(y \sigma_{1}\right) \sigma \lambda={ }_{E}$ $\left(y \sigma_{1}\right) \lambda_{1} \pi^{-1}={ }_{E} y \tau_{1} \pi^{-1}=(y \tau)^{\pi_{1}} \pi^{-1}=y \tau$ as required.

Finally, assume that $x$ is a variable occurring in $\Gamma_{0}$, but $x \notin Y_{1} \cup Y_{2}$. This means that $x$ has been substituted by a variable $y \in Y_{1} \cup Y_{2}$ during the variable identification step of the algorithm. On the one hand, this means that $y \tau=x \tau$ (since this must have triggered the identification). On the other hand, because of this identification step, we have defined $x \sigma:=y \sigma$. Thus we have $x \tau=y \tau={ }_{E} y \sigma \lambda=x \sigma \lambda$. This completes the proof of the proposition.

\section{Solving unification problems with constant restriction}

We have seen that algorithms for $E$-unification with linear constant restriction may be used to obtain - via our combination method-algorithms for general unification. In the first part of this section we shall describe how, conversely, algorithms for general unification can be used to solve unification problems with linear constant restrictions. In the second part, constant elimination algorithms together with algorithms for unification with constants are used to solve unification problems with arbitrary constant restriction. In the following, $F$ is assumed to be an arbitrary consistent equational theory. 


\subsection{Using algorithms for general unification}

In this subsection we shall consider both the problem of deciding solvability and of generating complete sets of solutions of unification problems with linear constant restrictions.

\section{The decision problem}

Let $\Gamma$ be an $F$-unification problem with a linear constant restriction, and let $<$ be the linear ordering by which this restriction is induced. In the following, let $X$ denote the set of all variables and $C$ denote the set of all free constants occurring in $\Gamma$. Our goal is to construct a general $F$-unification problem $\Gamma^{\prime}$ such that $\Gamma$ is solvable iff $\Gamma^{\prime}$ is solvable.

In this new system $\Gamma^{\prime}$, the free constants of $\Gamma$ will be treated as variables, i.e., the solutions are allowed to substitute terms for these "constants." For any free constant $c$ of $\Gamma$ we introduce a new (free) function symbol $f_{c}$ of arity $\left|V_{c}\right|$. Recall that $V_{c}=\{x \in X \mid x<c\}$ is the set of variables in whose $\sigma$-image $c$ must not occur for a solution $\sigma$ of the problem $\Gamma$. The general $F$-unification problem - in which the free constants of $\Gamma$ are treated as variables-is now defined as

$$
\Gamma^{\prime}:=\Gamma \cup\left\{c \doteq f_{c}\left(x_{1}, \ldots, x_{n}\right) \mid c \in C \text { and } V_{c}=\left\{x_{1}, \ldots, x_{n}\right\}\right\} .
$$

Proposition 5.1 The F-unification problem with linear constant restriction, $\Gamma$, is solvable iff the general $F$-unification problem $\Gamma^{\prime}$ is solvable.

Please note that the proposition only holds for unification problems with linear constant restriction. The following example demonstrates that the construction described above cannot be used for unification problems with arbitrary constant restriction.

Example 5.2 Let $F$ be the empty theory, and let $x, y$ be variables and $c, d$ be free constants. We consider the following $F$-unification problem with constant restriction:

$$
\Gamma=\{x \doteq d, y \doteq c\}, \quad V_{c}=\{x\}, V_{d}=\{y\} .
$$

It is easy to see that the restriction cannot be induced by a linear ordering on $\{x, y, c, d\}$. Obviously, the problem has the solution $\{x \mapsto d, y \mapsto c\}$. 
The corresponding general $F$-unification problem is

$$
\Gamma^{\prime}=\left\{x \doteq d, y \doteq c, c \doteq f_{c}(x), d \doteq f_{d}(y)\right\}
$$

where $c, d$ are now treated as variables. It is easy to see that this problem does not have a solution.

However, as we shall prove below, our construction is correct for unification problems with linear constant restriction, $\Gamma$.

\section{Generating complete sets of solutions}

Now we shall describe how this construction can be used to get a finite complete set of solutions of $\Gamma$, provided that a finite complete set of $F$-unifiers of $\Gamma^{\prime}$ exists.

Let $R$ be the possibly infinite ordered-rewriting system which is obtained when applying unfailing completion to $F$. We assume that the simplification ordering used during the completion also takes the additional symbols $f_{c}$ and variables (which are however treated as constants by the ordering) out of a countable set $X_{0}$ of new variables into account. This means that we can apply $R$ to terms built out of symbols in the signature of $F$, the additional symbols $f_{c}$, and variables in $X_{0}$. Let $T_{\downarrow R}$ be the $R$-irreducible elements of the set of these terms.

Now we shall show how an element $\sigma^{\prime}$ of a complete set of $F$-unifiers of $\Gamma^{\prime}$ can be used to define a solution $\sigma$ of $\Gamma$. Without loss of generality we may assume that $\sigma^{\prime}$ is $R$-normalized on the variables occurring in $\Gamma^{\prime}$. In fact, for any substitution there exists an ${ }_{F} F$-equivalent substitution which is $R$-normalized on the variables occurring in $\Gamma^{\prime}$; and exchanging an element of a complete set of $F$-unifiers of $\Gamma^{\prime}$ by an $={ }_{F}$-equivalent substitution still leaves us with a complete set of $F$-unifiers of $\Gamma^{\prime}$.

Let $\pi$ be a bijection from $T_{\downarrow R}$ onto a set of variables $Y$. This bijection has to satisfy two conditions. First, $Y$ should contain all the free constants occurring in $\Gamma$ (which are treated as variables in $\Gamma^{\prime}$ ). Since $\sigma^{\prime}$ is assumed to be $R$-normalized on the variables occurring in $\Gamma^{\prime}$, we have that $c \sigma^{\prime}$ is $R$-irreducible for all these constants $c$. The second condition on $\pi$ is that $\pi\left(c \sigma^{\prime}\right)=c$ for these constants $c$. The two conditions are satisfiable because for $c \neq c^{\prime}$ we have $c \sigma^{\prime} \neq c^{\prime} \sigma^{\prime}$. In fact, since $\sigma^{\prime}$ solves $\Gamma^{\prime}$, we know that $c \sigma^{\prime}={ }_{F} f_{c}\left(x_{1} \sigma^{\prime}, \ldots, x_{n} \sigma^{\prime}\right)$ and $c^{\prime} \sigma^{\prime}={ }_{F} f_{c^{\prime}}\left(x_{1} \sigma^{\prime}, \ldots, x_{n} \sigma^{\prime}\right)$. But this implies that $c \sigma^{\prime}$ has $f_{c}$ as root symbol, and $c^{\prime} \sigma^{\prime}$ the different symbol $f_{c^{\prime}}$. 
As described in Section 4, the bijection $\pi$ induces a mapping $\pi_{1}$. To this purpose we treat the symbols of the signature of $F$ as 1-symbols and the symbols $f_{c}$ as 2 -symbols. The mapping $\pi_{1}$ is now used to define our solution $\sigma$ of $\Gamma$. For all variables $x$ occurring in $\Gamma$ we define $x \sigma:=\left(x \sigma^{\prime}\right)^{\pi_{1}}$. The constants $c$ of $\Gamma$ are really treated as constants by $\sigma$, i.e., $c \sigma=c$. However, note that $c=\left(c \sigma^{\prime}\right)^{\pi_{1}}$ holds, anyway.

Proposition 5.3 Let $C\left(\Gamma^{\prime}\right)$ be a complete set of $F$-unifiers of $\Gamma^{\prime}$, which are (without loss of generality) assumed to be $R$-normalized on the variables occurring in $\Gamma^{\prime}$. Then the set $C(\Gamma):=\left\{\sigma \mid \sigma^{\prime} \in C\left(\Gamma^{\prime}\right)\right\}$, where $\sigma$ is constructed out of $\sigma^{\prime}$ as described above, is a complete set of solutions of the F-unification problem with linear constant restriction, $\Gamma$.

Again, the proposition only holds for unification problems with linear constant restriction.

\section{Proof of Proposition 5.1}

Recall that $X$ denote the set of all variables and $C$ denote the set of all free constants occurring in $\Gamma$.

To prove the "only-if" direction, assume that $\sigma$ is a solution of $\Gamma$. Without loss of generality we may assume that for all $x \in X$ the variables occurring in $x \sigma$ are new variables (i.e., variables not contained in $X$ ), and that $\sigma$ is the identity on all variables $y \notin X$. We define a substitution $\sigma^{\prime}$ on $X \cup C$ (where the elements of $C$ are now treated as variables) by induction on the linear ordering $<$ which induces the constant restriction of $\Gamma$.

First, we consider the least element of $X \cup C$ with respect to $<$. If this is a variable $x \in X$, then for all $c \in C$ we have $x \in V_{c}$. This implies that $x \sigma$ does not contain any of these free constants, and we can define $x \sigma^{\prime}:=x \sigma$. If the least element of $X \cup C$ is a constant $c \in C$, then $V_{c}=\emptyset$. This means that $f_{c}$ is a constant symbol, and we define $c \sigma^{\prime}:=f_{c}$.

Now let $x$ be an arbitrary element of $X$, and let $c_{1}, \ldots, c_{m} \in C$ be the free constants occurring in $x \sigma$. Since $\sigma$ satisfies the constant restriction induced by the linear ordering, the constants $c_{1}, \ldots, c_{m}$ (which are treated as variables in $\Gamma^{\prime}$ ) have to be smaller than $x$. That means that we may assume by induction that $c_{1} \sigma^{\prime}, \ldots, c_{m} \sigma^{\prime}$ are already defined. The term $x \sigma^{\prime}$ is now obtained from $x \sigma$ by replacing the $c_{k}$ by $c_{k} \sigma^{\prime}(k=1, \ldots, m)$. 
Finally, let $c$ be an arbitrary element of $C$. By definition, the system $\Gamma^{\prime}$ contains the equation $c \doteq f_{c}\left(x_{1}, \ldots, x_{n}\right)$, where $x_{1}, \ldots, x_{n}$ are the elements of $X$ which are smaller than $c$ with respect to $<$. Thus we can assume by induction that $x_{1} \sigma^{\prime}, \ldots, x_{n} \sigma^{\prime}$ are already defined, and we set $c \sigma^{\prime}:=f_{c}\left(x_{1} \sigma^{\prime}, \ldots, x_{n} \sigma^{\prime}\right)$.

It remains to be shown that $\sigma^{\prime}$ is a solution of $\Gamma^{\prime}$. Obviously, the definition of $\sigma^{\prime}$ implies that it solves the equations $c \doteq f_{c}\left(x_{1}, \ldots, x_{n}\right)$ in $\Gamma^{\prime}$. Now let $s \doteq t$ be an equation of $\Gamma$. Since $\sigma$ solves $\Gamma$, we get $s \sigma=_{F} t \sigma$. In addition, it is easy to see that for all $c \in C$ we have $c \sigma^{\prime}=c \sigma \sigma^{\prime}$ and for all $x \in X$ we have $x \sigma^{\prime}=x \sigma \sigma^{\prime}$. But then $s \sigma^{\prime}=s \sigma \sigma^{\prime}={ }_{F} t \sigma \sigma^{\prime}=t \sigma^{\prime}$.

To prove the "if" direction, assume that $\sigma^{\prime}$ is a solution of $\Gamma^{\prime}$. Without loss of generality, we assume that $\sigma^{\prime}$ is $R$-normalized on the set $X \cup C$ of all variables occurring in $\Gamma^{\prime}$. As described above, $\sigma^{\prime}$ can be used to define a new substitution $\sigma$ as follows: For all variables $x$ occurring in $\Gamma$ one defines $x \sigma:=\left(x \sigma^{\prime}\right)^{\pi_{1}}$. We have to show that $\sigma$ solves $\Gamma$.

Let $s \doteq t$ be an equation of $\Gamma$. Since $\sigma^{\prime}$ solves $\Gamma^{\prime}$, we know that $s \sigma^{\prime}={ }_{F} t \sigma^{\prime}$. Now we can apply Lemma 4.1 to get $\left(s \sigma^{\prime}\right)^{\pi_{1}}={ }_{F}\left(t \sigma^{\prime}\right)^{\pi_{1}}$. Using the definition of $\sigma$ and the fact that the terms $s, t$ do not contain the symbols $f_{c}$, it is easy to see that $\left(s \sigma^{\prime}\right)^{\pi_{1}}=s \sigma$ and $\left(t \sigma^{\prime}\right)^{\pi_{1}}=t \sigma$. Thus $\sigma$ really solves the equation $s \doteq t$.

It remains to be shown that $\sigma$ satisfies the constant restriction. Let $c \in C$ be a free constant. Since $\sigma^{\prime}$ solves $\Gamma^{\prime}$, we know that $c \sigma^{\prime}={ }_{F} f_{c}\left(x_{1} \sigma^{\prime}, \ldots, x_{n} \sigma^{\prime}\right)$, where $\left\{x_{1}, \ldots, x_{n}\right\}=V_{c}$. In addition, $\sigma^{\prime}$ was assumed to be $R$-normalized on $X \cup C$, which implies that $c \sigma^{\prime}, x_{1} \sigma^{\prime}, \ldots, x_{n} \sigma^{\prime}$ are $R$-irreducible terms; and since the symbol $f_{c}$ does not occur in any rule of $R$, the term $f_{c}\left(x_{1} \sigma^{\prime}, \ldots, x_{n} \sigma^{\prime}\right)$ is also $R$-irreducible. Thus we have $c \sigma^{\prime}=f_{c}\left(x_{1} \sigma^{\prime}, \ldots, x_{n} \sigma^{\prime}\right)$, which shows that $c \sigma^{\prime}$ is not a subterm of any of the terms $x_{1} \sigma^{\prime}, \ldots, x_{n} \sigma^{\prime}$. But then $c$ cannot occur in $x_{i} \sigma=\left(x_{i} \sigma^{\prime}\right)^{\pi_{1}}(i=1, \ldots, n)$.

\section{Proof of Proposition 5.3}

Now we shall show that the set $C(\Gamma)$, as defined in the formulation of Proposition 5.3, is a complete set of solutions of $\Gamma$. In the second part of the proof of Proposition 5.1 we have already shown that the substitution $\sigma$ constructed from a solution $\sigma^{\prime}$ of $\Gamma^{\prime}$ is itself a solution of $\Gamma$. Thus $C(\Gamma)$ is a set of solutions of $\Gamma$. It remains to be shown that it is a complete set. As before, let $X$ denote the set of variables and $C$ the set of free constants occurring in $\Gamma$. Recall that the elements of $C$ are treated as variables in $\Gamma^{\prime}$. 
Let $\tau$ be a solution of $\Gamma$. Without loss of generality we assume that, for all $x \in X$, the variables occurring in $x \tau$ are elements of $X_{0}$. As shown in the first part of the proof of Proposition 5.1, $\tau$ can be used to define a solution $\tau^{\prime}$ of $\Gamma^{\prime}$. Because of our assumption on $\tau$, it is easy to see that for all $z \in X \cup C$, the variables occurring in $z \tau^{\prime}$ are elements of $X_{0}$.

Since $C\left(\Gamma^{\prime}\right)$ is a complete set of $F$-unifiers of $\Gamma^{\prime}$, there exists an element $\sigma^{\prime}$ of $C\left(\Gamma^{\prime}\right)$ and a substitution $\lambda^{\prime}$ such that $\tau^{\prime}={ }_{F} \sigma^{\prime} \lambda^{\prime}\langle X \cup C\rangle$. Let $\sigma$ be the element of $C(\Gamma)$ constructed out of $\sigma^{\prime}$. Our aim is to define a substitution $\lambda$ which satisfies $\tau={ }_{F} \sigma \lambda\langle X\rangle$. Let $Y_{0} \subseteq Y$ denote the set of all variables occurring in the terms $x \sigma$ for $x \in X$. These terms may also contain elements of $C$, which however have to be treated as constants by $\lambda$.

In the construction of $\sigma$ out of $\sigma^{\prime}$ we have used a bijection $\pi$ from $T_{\downarrow R}$ onto a set of variables $Y$ which contains $C$. In addition, this bijection had to satisfy $\pi\left(c \sigma^{\prime}\right)=c$ for all $c \in C$. The substitution $\sigma$ was then defined by $x \sigma:=\left(x \sigma^{\prime}\right)^{\pi_{1}}$ for all $x \in X$. In order to be able to reverse the construction of $\tau^{\prime}$ out of $\tau$ we shall now consider an analogous bijection $\mu$ from $T_{\downarrow R}$ onto $Y$. The condition on $\mu$ is that $\mu\left(\left(c \tau^{\prime}\right)_{\downarrow R}\right)=c$ for all $c \in C$. Here $\left(c \tau^{\prime}\right)_{\downarrow R}$ denotes the unique $R$-irreducible element of the $=_{F}$-class of $c \tau^{\prime}$. As an easy consequence of this condition together with the definition of $\tau^{\prime}$, we get that $x \tau=\left(x \tau^{\prime}\right)^{\mu_{1}}$ for all $x \in X$.

The substitution $\lambda$ is now defined on all $y \in Y_{0}$ as $y \lambda:=\left(y \pi^{-1} \lambda^{\prime}\right)^{\mu_{1}}$. To complete the proof of the proposition we have to show that $\tau={ }_{F} \sigma \lambda\langle X\rangle$.

In this proof we need a lemma which is a stronger version of Lemma 4.1 for the special case of the union of an arbitrary equational theory $F$ with a disjoint free theory.

Lemma 5.4 Let $s, t$ be terms built out of symbols in the signature of $F$, the additional symbols $f_{c}$, and variables in $X_{0}$. Then $s={ }_{F} t$ iff $s^{\mu_{1}}={ }_{F} t^{\mu_{1}}$.

Proof. (1) From $s^{\mu_{1}}={ }_{F} t^{\mu_{1}}$ we can deduce $s^{\mu_{1}} \mu^{-1}={ }_{F} t^{\mu_{1}} \mu^{-1}$, and we also have $s={ }_{F} s^{\mu_{1}} \mu^{-1}$ and $t={ }_{F} t^{\mu_{1}} \mu^{-1}$.

(2) Obviously, it is sufficient to prove the "only-if" direction for the case where $t$ is obtained from $s$ by one application of an identity of $F$. Thus assume that $g=d$ is an identity of $F, u$ is an occurrence in $s$, and $\tau$ is a substitution such that $s=s[u \leftarrow g \tau], t=s[u \leftarrow d \tau]$. If the occurrence $u$ is strictly below an occurrence of a free function symbol, then it is easy to see that $s^{\mu_{1}}=t^{\mu_{1}}$. Otherwise, we have $s^{\mu_{1}}=s^{\mu_{1}}\left[u \leftarrow(g \tau)^{\mu_{1}}\right]$ and $s^{\mu_{1}}=s^{\mu_{1}}\left[u \leftarrow(d \tau)^{\mu_{1}}\right]$. What remains to be shown is $(g \tau)^{\mu_{1}}={ }_{F}(d \tau)^{\mu_{1}}$, and this can be done as in the proof of Lemma 4.1. 
We can now continue with the proof of the proposition. For all $x \in X$ we have $x \tau^{\prime}={ }_{F} x \sigma^{\prime} \lambda^{\prime}=\left(x \sigma^{\prime}\right)^{\pi_{1}} \pi^{-1} \lambda^{\prime}=x \sigma \pi^{-1} \lambda^{\prime}$. But then the lemma yields $x \tau=\left(x \tau^{\prime}\right)^{\mu_{1}}={ }_{F}\left(x \sigma \pi^{-1} \lambda^{\prime}\right)^{\mu_{1}}$. It remains to be shown that $\left(x \sigma \pi^{-1} \lambda^{\prime}\right)^{\mu_{1}}={ }_{F}$ $x \sigma \lambda$.

For this purpose, we define a substitution $\left(\pi^{-1} \lambda^{\prime}\right)^{\mu_{1}}$ on $Y_{0} \cup C$ as follows: for all $z \in Y_{0} \cup C, z\left(\pi^{-1} \lambda^{\prime}\right)^{\mu_{1}}:=\left(z \pi^{-1} \lambda^{\prime}\right)^{\mu_{1}}$. Because the terms $x \sigma$ for $x \in X$ do not contain any of the free function symbols $f_{c}$, it is easy to see that $\left(x \sigma \pi^{-1} \lambda^{\prime}\right)^{\mu_{1}}=(x \sigma)\left(\pi^{-1} \lambda^{\prime}\right)^{\mu_{1}}$.

Obviously, the substitutions $\lambda$ and $\left(\pi^{-1} \lambda^{\prime}\right)^{\mu_{1}}$ coincide on $Y_{0}$, but for $c \in C$ we need not have $c\left(\pi^{-1} \lambda^{\prime}\right)^{\mu_{1}}=c$. However, we can show that $c\left(\pi^{-1} \lambda^{\prime}\right)^{\mu_{1}}={ }_{F} c$. In fact, $c \pi^{-1} \lambda^{\prime}=c \sigma^{\prime} \lambda^{\prime}={ }_{F} c \tau^{\prime}$, and thus the lemma yields $\left(c \pi^{-1} \lambda^{\prime}\right)^{\mu_{1}}={ }_{F}$ $\left(c \tau^{\prime}\right)^{\mu_{1}}$. But our assumption on $\mu$ yields $\left(c \tau^{\prime}\right)^{\mu_{1}}=c$. To sum up, we have just seen that $\lambda={ }_{F}\left(\pi^{-1} \lambda^{\prime}\right)^{\mu_{1}}\left\langle Y_{0} \cup C\right\rangle$, which implies $(x \sigma) \lambda={ }_{F}(x \sigma)\left(\pi^{-1} \lambda^{\prime}\right)^{\mu_{1}}$. This completes the proof of the proposition.

\subsection{Using algorithms for constant elimination and for unification with constants}

In this subsection we shall consider unification problems with arbitrary constant restrictions. It will be shown how to reduce solving this kind of problems to solving both unification problems with constants and constant elimination problems.

A constant elimination problem in the theory $F$ is a finite set $\Delta=$ $\left\{\left(c_{1}, t_{1}\right), \ldots,\left(c_{n}, t_{n}\right)\right\}$ where the $c_{i}$ 's are free constants (i.e., constant symbols not occurring in the signature of $F$ ) and the $t_{i}$ 's are terms (built over the signature of $F$, variables, and free constants). A solution to such a problem is called a constant eliminator. It is a substitution $\sigma$ such that for all $i, 1 \leq i \leq n$, there exists a term $t_{i}^{\prime}$ not containing the free constant $c_{i}$ with $t_{i}^{\prime}={ }_{F} t_{i} \sigma$. The notion complete set of constant eliminators is defined analogously to the notion complete set of unifiers.

Let $\Gamma$ be an $F$-unification problem with arbitrary constant restriction. The goal is to construct a complete set of solutions of this problem. In the first step, we just ignore the constant restriction, and solve $\Gamma$ as an ordinary $F$-unification problem with constants. Let $C(\Gamma)$ be a complete set of $F$-unifiers of this problem. In the second step, we define for all unifiers $\sigma \in C(\Gamma)$ a constant elimination problem $\Delta_{\sigma}$ as follows:

$$
\Delta_{\sigma}:=\left\{(c, x \sigma) \mid c \text { is a free constant in } \Gamma \text { and } x \in V_{c}\right\} .
$$


For all $\sigma \in C(\Gamma)$, let $C_{\sigma}$ be a complete set of solutions of the constant elimination problem $\Delta_{\sigma}$.

Before we can describe the complete set of solutions of the $F$-unification problem with constant restriction, $\Gamma$, we have to define a slightly modified composition " $\otimes$ " of substitutions. Let $\sigma$ be an element of $C(\Gamma)$, and let $\tau$ be a constant eliminator in $C_{\sigma}$. Without loss of generality we assume that $\tau$ is the identity on variables not occurring in $\Delta_{\sigma}$, and that the terms $y \tau$ for variables $y$ occurring in $\Delta_{\sigma}$ contain only new variables. In particular, we will need for technical reasons that they do not contain variables occurring in terms $x \sigma$ for variables $x$ occurring in $\Gamma$.

For a given variable $x$, let $\left\{c_{1}, \ldots, c_{n}\right\}$ be the set of all constants $c_{i}$ occurring in $\Gamma$ such that $x \in V_{c_{i}}$. If this set is empty (i.e., $n=0$,) we define $x(\sigma \otimes \tau):=x \sigma \tau$. Now assume that $n>0$. Obviously, we have $\left\{\left(c_{1}, x \sigma\right), \ldots,\left(c_{n}, x \sigma\right)\right\} \subseteq \Delta_{\sigma}$. Since $\tau$ is a solution of $\Delta_{\sigma}$, there exist terms $s_{1}, \ldots, s_{n}$ such that for all $i, 1 \leq i \leq n, x \sigma \tau={ }_{F} s_{i}$ and $c_{i}$ does not occur in $s_{i}$. It is easy to see that this also implies the existence of a single term $s$ such $c_{1}, \ldots, c_{n}$ do not occur in $s$ and $x \sigma \tau={ }_{F} s$. We define $x(\sigma \otimes \tau):=s$.

Proposition 5.5 The set

$$
\bigcup_{\sigma \in C(\Gamma)}\left\{\sigma \otimes \tau \mid \tau \in C_{\sigma}\right\}
$$

is a complete set of solutions of the $F$-unification problem with constant restriction, $\Gamma$.

Proof. First, we have to show that the elements $\sigma \otimes \tau$ of this set solve all the equations $s \doteq t$ of $\Gamma$. Since $\sigma$ is an $F$-unifier of $\Gamma$, we have $s \sigma={ }_{F} t \sigma$, which implies $s \sigma \tau={ }_{F} t \sigma \tau$. But the definition of $\sigma \otimes \tau$ was such that $\sigma \tau={ }_{F} \sigma \otimes \tau$, and thus we get $s(\sigma \otimes \tau)={ }_{F} t(\sigma \otimes \tau)$.

Second, we must prove that $\sigma \otimes \tau$ satisfies the constant restriction. Assume that $x \in V_{c}$. Then the constant elimination problem $\Delta_{\sigma}$ contains the tuple $(c, x \sigma)$. By definition of $\sigma \otimes \tau$, we get that $x(\sigma \otimes \tau)$ is a term $s$ not containing $c$.

Finally, it remains to be shown that the set is complete. Assume that $\theta$ is a solution of the $F$-unification problem with constant restriction, $\Gamma$. In particular, this means that $\theta$ solves the $F$-unification problem $\Gamma$ (where the restrictions are ignored). Hence there exist an element $\sigma$ of the complete set $C(\Gamma)$ and a substitution $\lambda$ such that $\theta={ }_{F} \sigma \lambda\langle X\rangle$, where $X$ denotes the set of all variables occurring in $\Gamma$. Thus we have 
- for all $x \in X: x \theta={ }_{F}(x \sigma) \lambda$, and

- for all $c$ with $x \in V_{c}: c$ does not occur in $x \theta$,

which shows that $\lambda$ solves the constant elimination problem $\Delta_{\sigma}$. Consequently, there exist an element $\tau$ of the complete set $C_{\sigma}$ and a substitution $\lambda^{\prime}$ such that $\lambda={ }_{F} \tau \lambda^{\prime}\langle Y\rangle$, where $Y$ denotes the set of all variables occurring in $\Delta_{\sigma}$. Without loss of generality, we assume that $z \lambda^{\prime}=z \lambda$ for all variables $z$ not occurring in one of the terms $y \tau$ with $y \in Y$.

We want to show that for all $x \in X$ we have $x \theta={ }_{F} x(\sigma \otimes \tau) \lambda^{\prime}$. For all $x \in X$, we know that $x \theta={ }_{F} x \sigma \lambda$, and since $\sigma \tau={ }_{F} \sigma \otimes \tau$ we also have $x(\sigma \otimes \tau) \lambda^{\prime}={ }_{F} x \sigma \tau \lambda^{\prime}$. Thus it remains to be shown that $x \sigma \lambda={ }_{F} x \sigma \tau \lambda^{\prime}$. We have to distinguish two cases. First, assume that $(c, x \sigma) \in \Delta_{\sigma}$ for some $c$. In this case all variables occurring in $x \sigma$ are elements of $Y$, and thus $\lambda={ }_{F}$ $\tau \lambda^{\prime}\langle Y\rangle$ yields $x \sigma \lambda={ }_{F} x \sigma \tau \lambda^{\prime}$. For the second case, assume that $x \sigma$ contains a variable $z$ which is not an element of $Y$, the set of all variables occurring in $\Delta_{\sigma}$. We are finished if we can show that, nevertheless, $z \lambda={ }_{F} z \tau \lambda^{\prime}$ holds for all such variables $z$. Since $\tau$ was assumed to be the identity on variables not occurring in $\Delta_{\sigma}$, we have $z \tau=z$. Since $z$ occurs in $x \sigma$ for a variable $x \in X$, our second assumption on $\tau$ implies that $z$ does not occur in any term $y \tau$ with $y \in Y$. But then $z \lambda^{\prime}=z \lambda$ by our assumption on $\lambda^{\prime}$, which completes the proof of $x \sigma \lambda={ }_{F} x \sigma \tau \lambda^{\prime}$.

\section{Conclusion}

We have presented a new method for treating the problem of unification in the union of disjoint equational theories. Unlike most of the other methods developed for this purpose, it can be used to combine decision procedures as well as procedures computing finite complete sets of unifiers. Applicability of our method depends on a new type of prerequisite, namely on the solvability of unification problems with linear constant restrictions. Presupposing the existence of a constant elimination algorithm - as necessary for the method of Schmidt-Schauß - seems to be a stronger requirement. In fact, we have seen that constant elimination procedures can be used to solve unification problems with arbitrary constant restrictions. However, it is still an open problem whether there exists an equational theory for which solving unification problems with linear constant restrictions is finitary (or decidable) but solving unification problems with arbitrary constant restrictions is not. 
Our main results together with the results described in the previous section show that there is a close correspondence between solving unification problems with linear constant restrictions and solving general unification problems. For a given equational theory, the first kind of problems is decidable (finitary solvable) if and only if the second kind of problems is. As an interesting open problem it remains to be shown whether there exists an equational theory for which unification with constants is decidable (finitary) but general unification - or equivalently, solving unification problems with linear constant restrictions - is not. One should note that there already exist such results for the case of single equations, i.e., unification problems of cardinality one. Narendran and Otto [NO90] have shown that there exists an equational theory $E$ such that solvability is decidable for $E$-unification problems (with constants) of cardinality one, but is undecidable for $E$-unification problems of cardinality greater than one, and thus also for general $E$-unification problems.

To make the presentation and the proof of correctness of the combination method more concise, we did not consider possible optimizations which would rule out certain partitions in Step 3 and certain linear orderings in Step 4 of the algorithm.

\section{References}

[Ba91] F. Baader, "Unification Theory," Proceedings of the First International Workshop on Word Equations and Related Topics, to appear as Springer LNCS.

[Bc87] L. Bachmair, Proof Methods for Equational Theories, Ph.D. Thesis, Dept. of Comp. Sci., University of Illinois at Urbana-Champaign, 1987.

[Bo90] A. Boudet, "Unification in a Combination of Equational Theories: An Efficient Algorithm," Proceedings of the 10th International Conference on Automated Deduction, LNCS 449, 1990.

[BJ89] A. Boudet, J.P. Jouannaud, M. Schmidt-Schauß, "Unification in Boolean Rings and Abelian Groups," J. Symbolic Computation 8, 1989.

[Bü86] H.-J. Bürckert, "Some Relationships Between Unification, Restricted Unification, and Matching," Proceedings of the 8th International Conference on Automated Deduction, LNCS 230, 1986. 
[Bü90] H.-J. Bürckert, "A Resolution Principle for Clauses with Constraints," Proceedings of the 10th International Conference on Automated Deduction, LNCS 449, 1990.

[Co90] A. Colmerauer, "An Introduction to PROLOG III," C. ACM 33, 1990 .

[DJ87] N. Dershowitz, J.P. Jouannaud, "Rewrite Systems," Unité Associée au CNRS UA 410: AL KHOWARIZMI, Rapport de Recherche $n^{\circ}$ 478, 1989. To appear in Volume B of "Handbook of Theoretical Computer Science," North-Holland.

[Fa84] F. Fages, "Associative-Commutative Unification," Proceedings of the 7th International Conference on Automated Deduction, LNCS 170, 1984 .

[He86] A. Herold, "Combination of Unification Algorithms," Proceedings of the 8th International Conference on Automated Deduction, LNCS 230, 1986 .

[He87] A. Herold, Combination of Unification Algorithms in Equational Theories, Dissertation, Fachbereich Informatik, Universität Kaiserslautern, 1987.

[HS87] A. Herold, J.H. Siekmann, "Unification in Abelian Semigroups," J. Automated Reasoning 3, 1987.

[Ho76] J.M. Howie, An Introduction to Semigroup Theory, London: Academic Press, 1976.

[JL84] J. Jaffar, J.L. Lassez, M. Maher, "A Theory of Complete Logic Programs with Equality," J. Logic Programming 1, 1984.

[JL87] J. Jaffar, J.L. Lassez, "Constraint Logic Programming," Proceedings of 14th POPL Conference, Munich, 1987.

[JK86] J.P. Jouannaud, H. Kirchner, "Completion of a Set of Rules Modulo a Set of Equations," SIAM J. Computing 15, 1986.

[JK90] J.P. Jouannaud, C. Kirchner, "Solving Equations in Abstract Algebras: A Rule-Based Survey of Unification," Preprint, 1990. To appear in the Festschrift to Alan Robinson's birthday.

[KN91] D. Kapur, P. Narendran, "Complexity of Unification Problems with Associative-Commutative Operators," Preprint, 1991. To appear in J. Automated Reasoning. 
[Ki85] C. Kirchner, Méthodes et Outils de Conception Systématique d'Algorithmes d'Unification dans les Théories equationnelles, Thèse d'Etat, Univ. Nancy, France, 1985.

[KK89] C. Kirchner, H. Kirchner, "Constrained Equational Reasoning," Proceedings of SIGSAM 1989 International Symposium on Symbolic and Algebraic Computation, ACM Press, 1989.

[LS75] M. Livesey, J.H. Siekmann, "Unification of AC-Terms (bags) and ACI-Terms (sets)," Internal Report, University of Essex, 1975, and Technical Report 3-76, Universität Karlsruhe, 1976.

[Ma77] G.S. Makanin, "The Problem of Solvability of Equations in a Free Semigroup," Mat. USSR Sbornik 32, 1977.

[NO90] P. Narendran, F. Otto, "Some Results on Equational Unification," Proceedings of the 10th Conference on Automated Deduction, LNCS 449, 1990.

[P172] G. Plotkin, "Building in Equational Theories," Machine Intelligence 7, 1972 .

[Sc89] M. Schmidt-Schauß, "Combination of Unification Algorithms," J. Symbolic Computation 8, 1989.

[Sh91] K. Schulz, "Makanin's Algorithm - Two Improvements and a Generalization," CIS-Report 91-39, CIS, University of Munich, 1991.

[Si89] J.H. Siekmann, "Unification Theory: A Survey," in C. Kirchner (ed.), Special Issue on Unification, Journal of Symbolic Computation $\mathbf{7}, 1989$.

[St75] M. Stickel, "A Complete Unification Algorithm for AssociativeCommutative Functions," Proceedings of the International Joint Conference on Artificial Intelligence, 1975.

[St81] M. Stickel, "A Unification Algorithm for Associative-Commutative Functions," J. ACM 28, 1981.

[St85] M. Stickel, "Automated Deduction by Theory Resolution," J. Automated Reasoning 1, 1985.

[Ti86] E. Tiden, "Unification in Combinations of Collapse Free Theories with Disjoint Sets of Function Symbols," Proceedings of the 8th International Conference on Automated Deduction, LNCS 230, 1986. 
[Ye87] K. Yelick, "Unification in Combinations of Collapse Free Regular Theories," J. Symbolic Computation 3, 1987. 


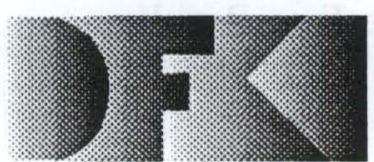

Deutsches

Forschungszentrum

für Künstliche

Intelligenz $\mathrm{GmbH}$

\section{DFKI Publikationen}

Die folgenden DFKI Veröffentlichungen sowie die aktuelle Liste von allen bisher erschienenen Publikationen können von der oben angegebenen Adresse bezogen werden.

Die Berichte werden, wenn nicht anders gekennzeichnet, kostenlos abgegeben.

\section{DFKI Research Reports}

R R-90-03

Andreas Dengel, Nelson M. Mattos: Integration of Document Representation, Processing and Management

18 pages

\section{R R-90-04}

Bernhard Hollunder, Werner Nutt: Subsumption

34 pages

\section{R R-90-05}

Franz Baader: A Formal Definition for the Expressive Power of Knowledge Representation Languages

22 pages

\section{R R-90-06}

Bernhard Hollunder: Hybrid Inferences in KL-ONEbased Knowledge Representation Systems

21 pages

\section{R R -90-07}

Elisabeth André, Thomas Rist: Wissensbasierte Informationspräsentation:

Zwei Beiträge zum Fachgespräch Graphik und KI:

1. Ein planbasierter Ansatz zur Synthese illustrierter Dokumente

2. Wissensbasierte Perspektivenwahl für die automatische Erzeugung von 3D-

24 Seiten Objektdarstellungen

R R-90-08

Andreas Dengel: A Step Towards Understanding Paper Documents

25 pages

R R-90-09

Susanne Biundo: Plan Generation Using a Method of Deductive Program Synthesis

17 pages Algorithms for Concept Languages
DFKI

-Bibliothek-

PF 2080

6750 Kaiserslautern

FRG

\section{DFKI Publications}

The following DFKI publications or the list of all publisched papers so far can be ordered from the above address.

The reports are distributed free of charge except if otherwise indicated.

\section{R R -90-10}

Franz Baader, Hans-Jürgen Bürckert, Bernhard Hollunder, Werner Nutt, Jörg H. Siekmann:

Concept Logics

26 pages

\section{R R-90-11}

Elisabeth André, Thomas Rist: Towards a PlanBased Synthesis of Illustrated Documents 14 pages

\section{R R-90-12}

Harold Boley: Declarative Operations on Nets 43 pages

\section{R R-90-13}

Franz Baader: Augmenting Concept Languages by Transitive Closure of Roles: An Alternative to Terminological Cycles 40 pages

\section{R R -90-14}

Franz Schmalhofer, Otto Kühn, Gabriele Schmidt: Integrated Knowledge Acquisition from Text, Previously Solved Cases, and Expert Memories 20 pages

\section{R R -90-15}

Harald Trost: The Application of Two-level Morphology to Non-concatenative German Morphology 13 pages

\section{RR-90-16}

Franz Baader, Werner Nutt: Adding Homomorphisms to Commutative/Monoidal Theories, or: How Algebra Can Help in Equational Unification 25 pages

\section{R R -90 - 17}

Stephan Busemann: Generalisierte Phasenstrukturgrammatiken und ihre Verwendung zur maschinellen Sprachverarbeitung 114 Seiten 


\section{R R-91-01}

Franz Baader, Hans-Jürgen Bürckert, Bernhard Nebel, Werner Nutt, Gert Smolka: On the Expressivity of Feature Logics with Negation, Functional Uncertainty, and Sort Equations 20 pages

\section{R R-91-02}

Francesco Donini, Bernhard Hollunder, Maurizio Lenzerini, Alberto Marchetti Spaccamela, Daniele Nardi, Werner Nutt: The Complexity of Existential Quantification in Concept Languages

22 pages

\section{RR-91-03}

B.Hollunder, Franz Baader: Qualifying Number Restrictions in Concept Languages

34 pages

\section{RR-91-04}

Harald Trost: X2MORF: A Morphological Component Based on Augmented Two-Level Morphology

19 pages

\section{RR-91-05}

Wolfgang Wahlster, Elisabeth André, Winfried Graf, Thomas Rist: Designing Illustrated Texts: How Language Production is Influenced by

Graphics Generation.

17 pages

\section{RR-91-06}

Elisabeth André, Thomas Rist: Synthesizing Illustrated Documents A Plan-Based Approach 11 pages

\section{R R-91-07}

Günter Neumann, Wolfgang Finkler: A HeadDriven Approach to Incremental and Parallel Generation of Syntactic Structures 13 pages

\section{R R-91-08}

Wolfgang Wahlster, Elisabeth André, Som Bandyopadhyay, Winfried Graf, Thomas Rist: WIP: The Coordinated Generation of Multimodal Presentations from a Common Representation 23 pages

RR - 91-09

Hans-Jürgen Bürckert, Jürgen Müller,

Achim Schupeta: RATMAN and its Relation to

Other Multi-Agent Testbeds

31 pages

RR - 91-10

Franz Baader, Philipp Hanschke: A Scheme for Integrating Concrete Domains into Concept Languages

31 pages
R R -91-11

Bernhard Nebel: Belief Revision and Default Reasoning: Syntax-Based Approaches 37 pages

R R -91-12

J.Mark Gawron, John Nerbonne, Stanley Peters: The Absorption Principle and E-Type Anaphora 33 pages

\section{R R -91-13}

Gert Smolka: Residuation and Guarded Rules for Constraint Logic Programming

17 pages

\section{R R - 91-14}

Peter Breuer, Jürgen Müller: A Two Level Representation for Spatial Relations, Part I 27 pages

\section{R R -91-15}

Bernhard Nebel, Gert Smolka: Attributive Description Formalisms ... and the Rest of the World

20 pages

\section{R R-91-16}

Stephan Busemann: Using Pattern-Action Rules for the Generation of GPSG Structures from Separate Semantic Representations

18 pages

\section{R R -91-17}

Andreas Dengel, Nelson M. Mattos:

The Use of Abstraction Concepts for Representing and Structuring Documents

17 pages

\section{R R -91-18}

John Nerbonne, Klaus Netter, Abdel Kader Diagne, Ludwig Dickmann, Judith Klein:

A Diagnostic Tool for German Syntax

20 pages

\section{RR -91-19}

Munindar P. Singh: On the Commitments and Precommitments of Limited Agents 15 pages

\section{R R -91-20}

Christoph Klauck, Ansgar Bernardi, Ralf Legleitner FEAT-Rep: Representing Features in CAD/CAM 48 pages

\section{R R -91-21}

Klaus Netter: Clause Union and Verb Raising Phenomena in German 38 pages 
RR-91-22

Andreas Dengel: Self-Adapting Structuring and Representation of Space

27 pages

RR-91-23

Michael Richter, Ansgar Bernardi, Christoph

Klauck, Ralf Legleitner: Akquisition und

Repräsentation von technischem Wissen für

Planungsaufgaben im Bereich der Fertigungstechnik

24 Seiten

R R-91-24

Jochen Heinsohn: A Hybrid Approach for Modeling Uncertainty in Terminological Logics

22 pages

RR - 91-25

Karin Harbusch, Wolfgang Finkler, Anne Schauder: Incremental Syntax Gencration with Trec Adjoining Grammars

16 pages

RR-91-26

M. Bauer, S. Biundo, D. Dengler, M. Hecking,

J. Koehler, G. Merziger:

Integrated Plan Generation and Recognition - A Logic-Based Approach -

17 pages

R R - 91 - 27

A. Bernardi, H. Boley, Ph. Ilanschke,

K. Hinkelmann, Ch. Klauck, O. Kühn,

R. Legleitner, M. Meyer, M. M. Richter,

F. Schmalhofer, G. Schmidt, W. Sommer:

ARC-TEC: Acquisition, Representation and

Compilation of Technical Knowledge

18 pages

\section{R R-91-28}

Rolf Backofen, Harald Trost, Ilans Uszkoreit:

Linking Typed Feature Formalisms and

Terminological Knowledge Representation

Languages in Natural Language Front-Ends

11 pages

R R-91-29

Hans Uszkoreit: Strategies for Adding Control

Information to Declarative Grammars

17 pages

R R -91-30

Dan Flickinger, John Nerbonne:

Inheritance and Complementation: A Casc Study of Easy Adjectives and Related Nouns

39 pages

R R-91-31

H.-U. Krieger, J. Nerbonne:

Feature-Based Inheritance Nctworks for

Computational Lexicons

11 pages
RR-91-32

Rolf Backofen, Lutz Euler, Günther Görz:

Towards the Integration of Functions, Relations and Types in an AI Programming Language

14 pages

R R -91-33

Franz Baader, Klaus Schulz:

Unification in the Union of Disjoint Equational

Theories: Combining Decision Procedures

33 pages

\section{R R -91-35}

Winfried Graf, Wolfgang Maaß: Constraint-basierte Verarbeitung graphischen Wissens

14 Seiten

\section{DFKI Technical Memos}

TM-90-03

Franz Baader, Bernhard Hollunder: KRIS:

Knowledge Representation and Inference System

-System Description-

15 pages

\section{TM-90-04}

Franz Baader, Hans-Jürgen Bürckert, Jochen Heinsohn, Bernhard Hollunder, Jürgen Müller, Bernhard Nebel, Werner Nutt, Hans-Jürgen Profitlich: Terminological Knowledge Representation: A Proposal for a Terminological Logic 7 pages

T M-91-01

Jana Köhler: Approaches to the Reuse of Plan Schemata in Planning Formalisms 52 pages

TM-91-02

Knut Hinkelmann: Bidirectional Reasoning of Horn Clause Programs: Transformation and Compilation 20 pages

T M-91-03

Otto Kühn, Marc Linster, Gabriele Schmidt:

Clamping, COKAM, KADS, and OMOS:

The Construction and Operationalization of a KADS Conceptual Model

20 pages

\section{TM-91-04}

Harold Boley (Ed.):

A sampler of Relational/Functional Definitions 12 pages

\section{T:M-91-05}

Jay C. Weber, Andreas Dengel, Rainer Bleisinger: Theoretical Consideration of Goal Recognition Aspects for Understanding Information in Business Letters

10 pages 
TM-91-06

Johannes Stein: Aspects of Cooperating Agents 22 pages

TM-91-08

Munindar P. Singh: Social and Psychological

Commitments in Multiagent Systems

11 pages

TM-91-09

Munindar P. Singh: On the Semantics of Protocols Among Distributed Intelligent Agents

18 pages

TM-91-10

Béla Buschauer, Peter Poller, Anne Schauder, Karin Harbusch: Tree Adjoining Grammars mit

Unifikation

149 pages

\section{TM-91-11}

Peter Wazinski: Generating Spatial Descriptions for Cross-modal References

21 pages

TM-91-12

Klaus Becker, Christoph Klauck, Johannes Schwagereit: FEAT-PATR: Eine Erwcitcrung des D-PATR zur Feature-Erkennung in CAD/CAM 33 Seiten

TM-91-13

Knut Hinkelmann:

Forward Logic Evaluation: Developing a Compiler from a Partially Evaluated Meta Interpreter 16 pages

\section{DFKI Documents}

\section{D-90-05}

Ansgar Bernardi, Christoph Klauck, Ralf

Legleitner: Formalismus zur Repräsentation von Geo-metrie- und Technologieinformationen als Teil eines Wissensbasierten Produktmodells 66 Seiten

\section{D-90-06}

Andreas Becker: The Window Tool Kit 66 Seiten

D-91-01

Werner Stein, Michael Sintek: Relfun/X - An Experimental Prolog Implementation of Relfun 48 pages

D-91-03

Harold Boley, Klaus Elsbernd, Hans-Günther Hein, Thomas Krause: RFM Manual: Compiling RELFUN into the Relational/Functional Machine 43 pages
D-91-04

DFKI Wissenschaftlich-Technischer Jahresbericht 1990

93 Seiten

\section{D-91.06}

Gerd Kamp: Entwurf, vergleichende Beschreibung und Integration eines Arbeitsplanerstellungssystems für Drehteile

130 Seiten

D-91-07

Ansgar Bernardi, Christoph Klauck, Ralf Legleitner TEC-REP: Repräsentation von Geometrie- und

Technologieinformationen

70 Seiten

D-91-08

Thomas Krause: Globale Datenflußanalyse und horizontale Compilation der relational-funktionalen Sprache RELFUN

137 pages

D-91-09

David Powers and Lary Reeker (Eds):

Proceedings MLNLO'91 - Machine Learning of

Natural Language and Ontology

211 pages

Note: This document is available only for a nominal charge of 25 DM (or 15 US-\$).

D - $91-10$

Donald R. Steiner, Jürgen Müller (Eds.):

MAAMAW'91: Pre-Proceedings of the 3rd

European Workshop on „Modeling Autonomous

Agents and Multi-Agent Worlds"

246 pages

Note: This document is available only for a nominal charge of $25 \mathrm{DM}$ (or 15 US-\$).

D - $91-11$

Thilo C. Horstmann:Distributed Truth Maintenance

61 pages

D-91-12

Bernd Bachmann:

$\mathrm{H}^{\text {icra }} \mathrm{C}_{\text {on }}$ - a Knowledge Representation System

with Typed Hierarchies and Constraints

75 pages

\section{D-91-13}

International Workshop on Terminological Logics

Organizers: Bernhard Nebel, Christof Peltason, Kai von Luck

131 pages

D-91-14

Erich Achilles, Bernhard Hollunder, Armin Laux, Jörg-Peter Mohren: XXRIS : Xnowledge

Representation and Inference System

- Benuzerhandbuch -

28 Seiten 


Franz Baader, Klaus Schulz 